\title{
Article
}

\section{Nonlinear Valuation with XVAs: Two Converging Approaches}

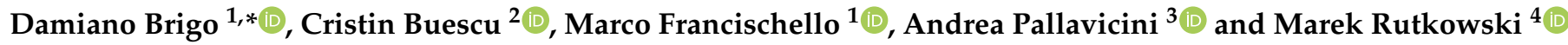 \\ 1 Department of Mathematics, Imperial College London, London SW7 2AZ, UK; \\ m.francischello14@imperial.ac.uk \\ 2 Department of Mathematics, King's College London, London WC2R 2LS, UK; cristin.buescu@kcl.ac.uk \\ 3 Financial Engineering, Intesa Sanpaolo Milan, 20121 Milan, Italy; andrea.pallavicini@intesasanpaolo.com \\ 4 School of Mathematics and Statistics, University of Sydney, Sydney 2006, Australia; \\ marek.rutkowski@sydney.edu.au \\ * Correspondence: damiano.brigo@imperial.ac.uk
}

check for updates

Citation: Brigo, D.; Buescu, C.; Francischello, M.; Pallavicini, A.; Rutkowski, M. Nonlinear Valuation with XVAs: Two Converging Approaches. Mathematics 2022, 10, 791. https://doi.org/10.3390/ math10050791

Academic Editors: Matthias Ehrhardt, Maria Do Rosário Grossinho and Daniel Sevcovic

Received: 14 December 2021 Accepted: 26 February 2022 Published: 2 March 2022

Publisher's Note: MDPI stays neutral with regard to jurisdictional claims in published maps and institutional affiliations.

Copyright: () 2022 by the authors. Licensee MDPI, Basel, Switzerland. This article is an open access article distributed under the terms and conditions of the Creative Commons Attribution (CC BY) license (https:// creativecommons.org/licenses/by/ $4.0 /)$.

\begin{abstract}
When pricing OTC contracts in the presence of additional risk factors and costs, such as credit risk and funding and collateral costs, the starting "clean price" is modified additively by valuation adjustments (XVAs) that account for each factor or cost in isolation, while seemingly ignoring the combined effects. Instead, risk factors and costs can be jointly accounted for ab initio in the pricing mechanism at the level of cash flows, and this "adjusted cash flow" approach leads to a nonlinear valuation formula. While for practitioners this made more sense because it showed which discount factor is used for which cash flow (recall the multi-curve environment post-crisis), for academics, the focus was on checking that the resulting nonlinear valuation formula is consistent with the theoretical arbitrage-free "replication approach" that we also analyse in the paper. We formulate specific reasonable assumptions, which ensure that the valuation formulae obtained by the two approaches coincide, thus reinforcing both academics' and practitioners' confidence in adopting such nonlinear valuation formulae in a multi-curve setup.
\end{abstract}

Keywords: risk-neutral valuation; replication; funding costs; default; collateral

MSC: 91G40; 60J28

\section{Introduction}

During the financial crisis, it became clear that the machinery for valuating contracts failed to account for credit risk, funding, and collateral costs. The first step to address this shortcoming was to adjust existing prices with nominally additive spread terms that accounted for each risk factor in isolation. Several of such additive valuation adjustments, which are collectively known as XVAs, were extensively studied in the existing literature and they are formally identified in our setup in Sections 2.3 and 3.3. The major issue here is the interplay between various costs and risks, which typically gives rise to inherently nonlinear features of non-classical, arbitrage-free pricing. It is thus clear that the tentative additive price decomposition is merely an approximation of an exact nonlinear solution. A rigorous theory of valuation in the presence of all such effects can be quite challenging. In general, it does not lead to an additive split in the above adjustments, but rather to all-encompassing nonlinear valuation paradigms that are based on more advanced mathematical tools, such as semi-linear PDEs or nonlinear BSDEs (see, e.g., [1-9]).

Recall that, in the classical approach, the valuation of a financial derivative can be carried out by taking to conditional expectation of discounted cash flows under the riskneutral probability measure $\mathbb{Q}^{r}$, which is defined by postulating that the price of the (non-dividend-paying) risky asset, $S$, discounted by the risk-free cash account, $B^{r}$, is a local martingale, where $B_{t}^{r}=e^{\int_{0}^{r} r_{u} d u}$. We will follow this approach in Section 2.1 when introducing the concept of the clean price of a contract, namely the price of the contract in the absence of default risk and with no funding and collateral costs. However, it should 
be emphasised that even in Section 3.2.1, our rationale is necessarily different from the classical case, since the process, $r$, can be seen as a theoretical risk-free rate, which is not necessarily available for trading and thus it cannot be used for financing hedging strategies. Notwithstanding, we find it convenient to use it as a benchmark rate for funding spreads, as well as a formal tool for a model calibration and, of course, the computation of the contract's abstract clean price. This argument is also consistent with the most recent developments in the area of fixed income derivatives and benchmark rate definitions, and thus a novel theoretical approach to pricing of derivatives is emerging (see, e.g., [10-16], as well as [17-21] for technical documents and market data).

Although this is by no means formally required in our approach, it is natural to give to the process, $r$, the financial interpretation of a hypothetical risk-free rate, that is, the short-term rate for lending/borrowing between entities, which are postulated to be completely devoid of credit risk. In practice, one can consider some market proxies for the risk-free rate. In recent years, new benchmark rates for secured and unsecured transactions emerged. Currently, in the European financial market, the euro short-term rate (ESTR) is the benchmark for overnight transaction and it can be seen as a proxy for the riskfree rate, since it is based on unsecured fixed-rate deposit transactions, but those which were conducted and settled on the previous business day, so that we could consider the counterparty risk contribution negligible. In the United States, the benchmark for overnight transactions is the secured overnight financing rate (SOFR), which is based on secured overnight transactions. In the United Kingdom, the benchmark rate is the sterling overnight index average (SONIA), and in Japan it is the Tokyo overnight average rate (TONAR), both based on unsecured transactions.

The adopted in practice additive XVA pricing approximation was significantly improved by the "adjusted cash flows" approach (see, e.g., $[2,3,22,23]$ ) that went to first principles and factored these risk factors and costs in the cash flows of the contract. The approach hinges on modifying the future cash flows of the contract by adding explicit cash contributions representing the additional risks and costs, and then taking risk-neutral expectation of the deflated adjusted cash flows. The resulting general master pricing equation with all effects was initially introduced in Pallavicini et al. [22,23], and the existence and uniqueness of solutions to such equations was rigorously studied, for example, in $[2,3]$. The "adjusted cash flows" approach extends the cash flows to which risk-neutral expectation applies, but such extensions do not necessarily result in additive adjustments to basic risk-neutral prices, in that, from a pathwise point of view, the adjusted cash flows depend on the future values of the trade itself.

A natural question that arises with this approach is whether this is consistent with the classic "replication approach", as presented, for instance, in [24], for the case of collateralization and funding costs, but without defaults (see also [25-28] for results on pricing and hedging of credit risk). It is entirely conceivable, without seeing an actual proof, that the two approaches result in two different valuation formulae that are not in accord.

The contribution of this paper is to answer the above question in the affirmative in the usual case. To explain what we mean by the usual case, recall that the "adjusted cash flow" approach incorporates external funding costs in its full generality, and these give rise to an external funding debit valuation adjustment (the DVA2 term in [29]) in the pricing formula. We consider only the usual case, where these additional external costs are not considered, and show that the two valuation formulae obtained by the two approaches are, in fact, one and the same. Furthermore, we show that, for claims that can be replicated, the explicit expression of the cash flows adjustments that need to be operated in the valuation is no longer the result of astute insights into the contract cash flows, but an immediate outcome of the replication approach.

In the present contribution, we do not perform numerical investigations, since they are carefully described in the papers previously cited, where the "adjusted cash flows" approach and the "replication approach" are introduced. In particular, in those papers, 
specific choices for the dynamics of interest rates and their correlations are selected and discussed, see for example [30,31].

We stress that the choice of a formal "risk-neutral probability" is in fact largely arbitrary. For practical reasons, it is common to focus on a proxy for the risk-free rate, which is not traded but is employed to compute clean prices and calibrate classical models implemented by the trading desk, such as the Black-Scholes model, the SABR model, various stochastic volatility models, or classical term structure models-such as Vasicek's models or market models of LIBOR and swap rates. A practical application of this nonlinear valuation formula is illustrated in [32], where all additional risk factors are integrated in the valuation from first principles, without forcing separate adjustments to the clean price. When applied to a specific vulnerable contract this valuation leads to the modified Black-Scholes option pricing formula with dividends, which in turn allows for efficient sensitivity analysis.

The paper is organized as follows. Section 2 presents the "adjusted cash flows" approach including the margining, close-out at time of default, and funding extensions. Such analysis shows clearly the nonlinear features affecting the valuation and discusses further under which conditions the valuation formula can be disentangled in a series of additive adjustments. For the derivation of the main result in that section, Proposition 1, we refer to [22], where sound financial arguments are used to identify additional costs and benefits and hence the associated pricing adjustments with respect to the (admittedly abstract) clean pricing.

Sections 3 and 4 are devoted to a fully rigorous derivation of the valuation equations under a replication approach with explicit funding accounts and suitable self-financing conditions, first in the linear case and then when nonlinear effects show up (e.g., under asymmetric funding rates for a hedge). The treatment provides formal proofs and generalizes a number of results previously obtained through the risk-neutral extended cash flows approach of Section 2. We also emphasize the connection between valuation adjustments and solutions to either linear (in Section 3) or nonlinear (in Section 4) backward stochastic differential equations (BSDEs) for the price and hedge of a defaultable collateralized contract in a multi-curve model.

Section 5 discusses the case of an incomplete market, where we relax the replication assumption that had been adopted implicitly in Section 2, and explicitly in Sections 3 and 4. For the incomplete case, we can still present a derivation that is reminiscent of the previous sections, but which holds under an alternative set of assumptions based on the idea that the extended market should remain arbitrage-free, and thus the investor's deflated gains process should be a martingale under some probability measure.

\section{Risk-Neutral Valuation with Adjusted Cash Flows}

Consider the position of an investor who enters an over-the-counter (OTC) derivative contract, $A$, with a counterparty in the market. The contract specifies, in particular, the promised cash flows from inception to maturity, dependent on the values of underlying assets. These cash flows form the basis of the initial valuation of the contract, which results in its clean price, by which we mean the price of the non-defaultable uncollateralized contract under the postulate that the hedge is funded at the risk-free rate. As is well known from the classic theory of arbitrage-free pricing, the clean price can be computed as a conditional expectation of discounted future cash flows under a risk-neutral probability measure. However, in the currently prevailing market environment, this step is only the starting point of the actual valuation of an OTC contract between default-prone counterparties, because additional factors, such as default events, close-out payoff, collateral (margin account), and funding costs, have significant implications on extended arbitrage-free valuation, leading to valuation adjustments (XVAs) to the clean price.

Instead of assuming that these adjustments are additive, the valuation approach developed in [2,3,23], with early precursors as [33] in the case of pure default risk, adjusts the cash flows for each additional consideration-default, collateral, funding costs, and so on-thus being referred to as the risk-neutral "adjusted cash flow" approach. In this 
section, we will revisit this approach, starting from the computation of the clean price of an OTC contract in the absence of other risk factors, and then reviewing the cash flows considered in the adjustment of the clean price due to collateralization, on-default close-out cash flows and funding cash flows.

We fix a finite trading horizon date, $T>0$, for our model of the financial market given on a filtered probability space, $(\Omega, \mathcal{G}, \mathbb{G}, \mathbb{P})$, where the filtration $\mathbb{G}=\left(\mathcal{G}_{t}\right)_{t \in[0, T]}$ satisfies the usual conditions of right-continuity and completeness, and has the trivial initial $\sigma$-field $\mathcal{G}_{0}$. We assume that all the processes to be introduced are implicitly $\mathbb{G}$-adapted and, as usual, any semimartingale is a càdlàg process.

\subsection{Clean Price of an OTC Contract}

As explained in the introduction, we will use the process, $r$, to model an abstract risk-free short-term interest rate, which typically cannot be used for trading and, in fact, only a proxy for that rate can be observed. Still, it is possible to proceed by formally defining the associated discount factor, $D(t, s)=D^{r}(t, s)$, on the time interval, $[t, s]$, by

$$
D^{r}(t, s):=e^{-\int_{t}^{s} r_{u} d u}=B_{t}^{r}\left(B_{s}^{r}\right)^{-1} .
$$

In order to value an OTC contract, we first introduce the conventional, but nevertheless practically important, notion of the contract's clean price. To this end, it suffices to focus on the promised cash flows of the contract and temporarily ignore all crucially important for valuation and hedging issues related to the funding of the trade and default risk of the investor and the counterparty.

Definition 1 (Non-defaultable, uncollateralized derivative). A non-defaultable and uncollateralized version of a given contract is formally specified by a $\mathbb{G}$-adapted, finite variation process, $\left(A_{t}\right)_{t \in[0, T]}$, which gives the cumulative stream of cash flows representing all future promised payoffs from a given contract with maturity, $T$, with $A_{0}=0$.

By definition, the process $A$ describes all the cash flows associated with a contract that is non-defaultable and uncollateralized, including a potential final payment but it needs to be complemented by additional cash flows if a contract is defaultable and collateralized. We stress that the initial value of the contract is not included in the cash flow stream $A$, since our goal is to derive the initial value from the future contract's cash flows, funding arrangements, and model inputs.

For instance, for a non-defaultable zero-coupon bond maturing at $T$ the cash flows are $A_{t}^{b}=\mathbb{1}_{\{t \geq T\}}$ for the buyer and $A_{t}^{s}=-\mathbb{1}_{\{t \geq T\}}$ for the seller of the bond. Similarly, for a European call option written on equity with price $S$, strike $K$, and maturity $T$, we have $A_{t}^{b}=\left(S_{T}-K\right)^{+} \mathbb{1}_{\{t \geq T\}}$ for the buyer and $A_{t}^{s}=-\left(S_{T}-K\right)^{+} \mathbb{1}_{\{t \geq T\}}$ for the seller of the option, where-for any real number $x$-we write $x=x^{+}-x^{-}$, where $x^{+}:=x \vee 0$ and $x^{-}:=(-x) \vee 0$. We refer to [3] (Appendix A) for the valuation of this call without credit risk.

The discounted contribution of the promised cash flows during the lifetime of the contract, from date $t$ onwards, is given by

$$
\Pi(t, A):=\int_{] t, T]} D(t, u) d A_{u}
$$

where the cash flows stream $A$ depends on whether one takes the side of the buyer or seller of the contract and thus it is equal to either $A^{b}$ (for the buy-side) or $A^{s}$ (for the sell-side). This distinction is crucial in the case of nonlinear pricing where solutions to unilateral pricing problems with the processes $A$ and $-A$ will typically yield prices with different absolute values after alignment so that the bid-ask spread will arise.

We stress once again that it is not postulated that the investor may either lend or borrow cash at the theoretical risk-free rate, $r$. The primary role of the abstract rate $r$ is to 
serve as a benchmark rate with respect to which various funding spreads are computed, as it is in fact carried out in practice. Nevertheless, we formally apply here the risk-neutral valuation approach and thus the process $r$ is used to define the martingale measure, $\mathbb{Q}^{r}$, for deflated prices of non-dividend-paying traded assets. In practical implementations of this approach, the martingale measure, $\mathbb{Q}^{r}$, is obtained through calibration of a model to market quotes for the most liquid (and, presumably, also default-free) contracts, such as overnight loans or centrally cleared trades with negligible default risk.

Definition 2 (Clean price). The clean price $\pi_{t}^{r}(A)$ of the uncollateralized and non-defaultable derivative is given by the risk-neutral expectation under $\mathbb{Q}^{r}$ of all discounted cash flows that occur after time, $t$, as follows:

$$
\pi_{t}^{r}(A):=\mathbb{E}_{\mathbb{Q}^{r}}\left(-\Pi(t, A) \mid \mathcal{G}_{t}\right)
$$

The role of the minus sign in (1) is to entail that negative cash flows in $A$ produce positive contributions to the clean price for the seller of a contract (and negative for its buyer), which is consistent with the idea of pricing via replication, also within a nonlinear setup. This convention should be contrasted with the mark-to-market convention at time $t$, which is formally given by $-\pi_{t}^{r}(A)$ so that it yields a positive value for the option holder and a negative value for its writer. The latter convention, which is consistent with risk management and accounting view (as opposed to trading view), was used in related papers $[2,22,23]$.

Intuitively, the clean price can be seen as a purely hypothetical value of the contract, which would be its true price if the rate $r$ was available for trading and the market was perfect, that is, with no counterparty credit risk, collateralization, differential funding costs, default events, etc. The classical arbitrage-free pricing theory is mainly devoted to clean pricing and, more recently, the term clean price was introduced in order to make a clear distinction between the classical simplified pricing and fully developed (either linear or nonlinear) pricing paradigms that account for market realities and thus are more adequate for practical purposes. The latter approaches are tailored to deal with all-inclusive selling/buying prices of a contract, which are also known as the exit price from the perspective of the sell-side (i.e., the price that would be received to sell an asset) and the entry price for the buy-side (i.e., the price that would be paid to buy an asset). In principle, the computation of the clean price is independent of a company and their counterparty, whereas the exit and entry prices depend on a company and a counterparty in a given trade and thus they are idiosyncratic, rather than universal for all market participants.

\subsection{Adjusted Price of a Defaultable Collateralized Contract}

We present now a derivative contract that is collateralized and defaultable, and consider the cash flows impacted by these risk factors, together with the funding situation, in order to adjust the valuation formula (and implicitly the "clean price") by incorporating the corresponding cash flows in the risk-neutral expectation. As is customary in the credit risk literature (see, e.g., $[28,34,35]$ ), we assume that the filtration $\mathbb{F}$ is generated by the price process, $S$, and the full filtration $\mathbb{G}$ is such that $\mathbb{F} \subset \mathbb{G}$ and the default times of the investor and the counterparty, $\tau_{I}$ and $\tau_{C}$, are $\mathbb{G}$-stopping times (but not necessarily $\mathbb{F}$-stopping times). For simplicity, we usually assume that $\mathbb{Q}^{r}\left(\tau_{I}=\tau_{C}\right)=0$, which indeed holds in most of the intensity-based models of default risk. To define a defaultable, collateralized contract, we first specify the factors which generate the price adjustments, and then present the resulting adjusted valuation formula.

Cost of collateralization. When default risk is present, it is customary to mitigate it by implementing a collateralization procedure, which is also known among practitioners as "margining". Let $C_{t}$ denote the level of the collateral account at time $t$, as specified by the credit support annex (CSA), which defines the terms for the provision of collateral by the parties in the contract. For conciseness, we do not differentiate between the initial and variation margins so $C_{t}$ represents the total collateralization. By convention, $C_{t}>0$ 
means that the collateral has been overall posted by the counterparty to protect the investor, and the investor has to pay instantaneous interest $c_{t}^{b}$ on the related amount. If $C_{t}<0$, then the investor posts collateral for the counterparty and is remunerated at interest $c_{t}^{l}$. Therefore, the effective collateral accrual rate $\bar{c}$ is given by $\bar{c}_{t}:=c_{t}^{b} \mathbb{1}_{\left\{C_{t}>0\right\}}+c_{t}^{l} \mathbb{1}_{\left\{C_{t}<0\right\}}$. Here, we assume the collateral account can be rehypothecated (see [30] for a discussion on how collateralization impacts the close-out specification). We denote the discounted net cash flows due to the variation margining procedure over $[t, s]$ by $\gamma(t, s, C)$, where

$$
\gamma(t, s, C)=\int_{t}^{s} D(t, u) C_{u}\left(\bar{c}_{u}-r_{u}\right) d u
$$

Note that if the collateral rates $c^{b}$ and $c^{l}$ are both equal to the risk-free rate $r$, then $\gamma(t, s, C)$ vanishes.

Close-out cash flows. The most significant manifestation of the credit default risk is the actual default itself, and we consider the cash flow to and from the investor at the first default. Once a default event occurs, the contract is terminated (we have close-out) and all payments that are accelerated and become due are calculated under the close out payoff. Contractual cash flows have been exchanged up until the arrival of the first default time, either that of the investor $I$ or that of the counterparty $C$. We define the first-to-default time $\tau$ as $\tau:=\tau_{C} \wedge \tau_{I}$, resulting in an effective maturity $\widehat{\tau}$ of the contract given by $\widehat{\tau}:=\tau \wedge T$. An important feature of a defaultable contract is the credit support annex (CSA) close-out payoff, which occurs when one of the parties defaults either before or at the maturity of the contract. To define the CSA close-out payoff $\vartheta_{\tau}(R, C)$ on the event $\{\tau \leq T\}$, we first define the random variable $Y=Q_{\tau}-C_{\tau-}$, where $Q$ is the CSA close-out valuation process of the contract inclusive of the increment $\Delta A_{\tau}=A_{\tau}-A_{\tau-}$, representing a (possibly null) promised bullet dividend at $\tau$ and $C_{\tau-}$ is the value of the collateral process $C$ at the moment of the first default. Since the margin account is not updated at the moment of the first default, it can be represented as $\widetilde{C}_{t}=\mathbb{1}_{\{t<\tau\}} C_{t}+\mathbb{1}_{\{t \geq \tau\}} C_{\tau-}$, so that $\widetilde{C}_{\tau}=\widetilde{C}_{\tau-}$.

We note that the "adjusted cash flows" approach includes both the risk-free close-out valuation process, which is given by

$$
Q_{t}=-\pi_{t}^{r}(A)+\Delta A_{t}=\mathbb{E}_{\mathbb{Q}^{r}}\left(\int_{[t, T]} D(t, u) d A_{u} \mid \mathcal{G}_{t}\right),
$$

and the replacement close-out (see Brigo, Buescu, and Morini [36] or Durand and Rutkowski [37])

$$
Q_{t}=\widetilde{V}_{t}+\Delta A_{t}
$$

where $\widetilde{V}$ is an $\mathbb{F}$-adapted process representing the pre-default marked-to-market price of the contract for the investor (see $[2,3,23]$ ). Notice that the left-open interval is always used when dealing with the usual ex-dividend price, that is, the price of all future cash flows that occur strictly after time $t$. In contrast, when computing the risk-free close-out valuation process, we follow the market convention that the cash flow that should have occurred at the moment of default is also accounted for in valuation. Hence we deal with the cum-dividend price and thus we need to use left-closed intervals when computing the close-out valuation process (see also Definition 12 in Section 3.3).

One may set $C_{t}=\alpha_{t} Q_{t}$, where $\alpha$ is some $\mathbb{F}$-adapted process taking values in $[0,1]$ and $Q$ is either the risk-free close-out (an exogenous collateral) or the replacement close-out (an endogenous collateral). We refer the reader to Section 3.1.3 in Crépey et al. [38] for more comments regarding the financial interpretation of the amount owed by either party at time of the first default and the origin of each term in Equation (3). Let $R_{I}$ and $R_{C}$ denote recovery coefficients for the investor and the counterparty, respectively. 
Definition 3 (Close-out payoff). The CSA close-out payoff $\vartheta_{\tau}(R, C):=R_{\tau}+C_{\tau-}$ on the event $\{\tau \leq T\}$ where the recovery payoff $R_{\tau}$ is given by the following expression:

$$
R_{\tau}:=\mathbb{1}_{\left\{\tau_{C}<\tau_{I}\right\}}\left(R_{C} \mathrm{Y}^{+}-\mathrm{Y}^{-}\right)+\mathbb{1}_{\left\{\tau_{I}<\tau_{C}\right\}}\left(\mathrm{Y}^{+}-R_{I} \mathrm{Y}^{-}\right)+\mathbb{1}_{\left\{\tau_{I}=\tau_{C}\right\}}\left(R_{C} \mathrm{Y}^{+}-R_{I} \mathrm{Y}^{-}\right) .
$$

Example 1. Let us examine the equality (3) from the perspective of the seller of an option. Then, the value of the risk-free (or replacement) close-out $Q_{t}$ is manifestly negative; hence the seller is always pledging collateral to the option holder. The case of full collateral means that $C_{t}=Q_{t}$ for every $t$, and thus, in particular, $\mathrm{Y}=0$. More generally, if $C_{t}=\alpha_{t} Q_{t}$ for some process $\alpha$ taking values in $[0,1]$, then $\mathrm{Y}=Q_{\tau}-\alpha_{\tau-} Q_{\tau-} \leq 0$, provided that $Q_{\tau-}=Q_{\tau}$. In that case, $\mathrm{Y}=-\mathrm{Y}^{-}$ and thus (3) simplifies to

$$
R_{\tau}:=\mathbb{1}_{\left\{\tau_{C}<\tau_{I}\right\}} \mathrm{Y}+\mathbb{1}_{\left\{\tau_{I} \leq \tau_{C}\right\}} R_{I} \mathrm{Y} .
$$

This means that, as expected, the counterparty credit risk is only reflected through their default time $\tau_{C}$ but not their recovery rate $R_{C}$.

Note that the last two terms in (3) can be dropped when computing this from the point of view of the shareholders who do not benefit from it. For implications on the MillerModigliani theorem we refer the reader to [39]. To identify various valuation adjustments caused by the counterparty credit risk, it is convenient to represent the close-out payoff as follows:

$$
\vartheta_{\tau}(R, C)=Q_{\tau}-\mathbb{1}_{\left\{\tau_{C}<\tau_{I}\right\}} L_{C} Y^{+}+\mathbb{1}_{\left\{\tau_{I}<\tau_{C}\right\}} L_{I} Y^{-}+\mathbb{1}_{\left\{\tau_{I}=\tau_{C}\right\}}\left(L_{I} Y^{-}-L_{C} Y^{+}\right)
$$

where $L_{C}=1-R_{C}$ (respectively, $L_{I}=1-R_{I}$ ) is the loss-given default coefficient for the counterparty (respectively, the investor). Obviously, the loss-given default coefficient equal to one (respectively, zero) corresponds to the case of null (respectively, full) recovery. On the one hand, when either $L_{C}=L_{I}=0$ or $C=Q$, then we obtain $\vartheta_{\tau}(R, C)=Q_{\tau}$, so indeed we deal here with the full recovery of the CSA close-out value $Q_{\tau}$ of the contract. On the other hand, due to the presence of collateral, in the case of the total loss-given default, that is, when $L_{C}=L_{I}=1$, the CSA close-out payoff to the bank equals the following:

$$
\begin{aligned}
\vartheta_{\tau}(R, C) & =\mathbb{1}_{\left\{\tau_{C}<\tau_{I}\right\}}\left(Q_{\tau} \mathbb{1}_{\left\{Q_{\tau}<C_{\tau-}\right\}}+C_{\tau-} \mathbb{1}_{\left\{Q_{\tau} \geq C_{\tau-}\right\}}\right) \\
& +\mathbb{1}_{\left\{\tau_{I}<\tau_{C}\right\}}\left(Q_{\tau} \mathbb{1}_{\left\{Q_{\tau} \geq C_{\tau-}\right\}}+C_{\tau-} \mathbb{1}_{\left\{Q_{\tau}<C_{\tau-}\right\}}\right)+\mathbb{1}_{\left\{\tau_{C}=\tau_{I}\right\}} C_{\tau-} .
\end{aligned}
$$

Funding costs and benefits. In this step, we focus on the funding costs for implementing the trading/hedging strategy and we add the relevant cash flows by adopting the procedure proposed in Pallavicini et al. [23]. Let $F_{t}$ be the cash account for the hedging of the trade and let $H_{t}$ stand for the value of the investor's positions in the underlying risky asset, $S$. We assume that $S$ can be traded through a repo (repurchase agreement) market, meaning that the risky asset, $S$, is funded using a cash account, $F_{t}^{S}$, and the equality $F_{t}^{S}=-H_{t}$ holds for every $t \in[0, T]$. The case of collateralized risky assets can be treated in the same way by interpreting the cash account as the collateral account for such assets. We assume there are two funding rates $-f^{b}$ for borrowing money and $f^{l}$ for lending money-and similarly two repo rates, $h^{b}$ and $h^{l}$. The funding policy of the bank's treasury is determined by funding rates for cost, $f^{b}$, and benefit, $f^{l}$, of carry of hedge accounts, which both depend on the funding policy of the bank.

Definition 4 (Defaultable, collateralized contract). A defaultable, collateralized contract $(A, R, C, \tau)$ is a contract with the promised cash flows process $A$, collateral account $C$, and close-out payoff $\vartheta_{\tau}(R, C)$ occurring at the first default time $\tau$.

The "adjusted cash flows" pricing formula for the contract specified in Definition 4 was derived in detail in Pallavicini et al. [22], who used financial reasoning to identify additional costs or benefits with respect to the clean pricing. In Proposition 1, we present 
without proof the version of the risk-neutral pricing formula obtained in Brigo et al. [3]. Notice that in Proposition 1 the processes $C$ and $Q$ are arbitrary but the promised cash flows process $A$ is stopped at $\tau-$. For extensions of Proposition 1 obtained by the replication approach, see Corollary 3 , where $Q$ is an arbitrary process, and Proposition 3 , where the close-out valuation is given by the contract's clean price $\pi^{r}(A)$. We write $\widetilde{A}_{t}:=$ $\mathbb{1}_{\{t<\tau\}} A_{t}+\mathbb{1}_{\{t \geq \tau\}} A_{\tau-}$ to denote the promised cash flows of $A$ that are stopped either just before the first default or at the maturity date $T$, whichever is sooner.

Proposition 1 ("Adjusted cash flows" pricing formula). The risk-neutral price of the defaultable, collateralized contract $(A, R, C, \tau)$ inclusive of funding costs is obtained as, on the event $\{t<\tau\}$ for every $t \in[0, T]$,

$$
\begin{aligned}
& \pi_{t}^{r, f, h}(A, R, C, \tau)=\mathbb{E}_{\mathbb{Q}^{r}}\left(-\int_{t}^{\widehat{\tau}} D(t, u) d \widetilde{A}_{u}+\int_{t}^{\widehat{\tau}} D(t, u) C_{u}\left(\bar{c}_{u}-r_{u}\right) d u \mid \mathcal{G}_{t}\right) \\
& +\mathbb{E}_{\mathbb{Q}^{r}}\left(-\mathbb{1}_{\{\tau \leq T\}} D(t, \tau) \vartheta_{\tau}(R, C)+\int_{t}^{\widehat{\tau}} D(t, u) F_{u}\left(r_{u}-\bar{f}_{u}\right) d u+\int_{t}^{\widehat{\tau}} D(t, u) F_{u}^{S}\left(r_{u}-\bar{h}_{u}\right) d u \mid \mathcal{G}_{t}\right) .
\end{aligned}
$$

It is important to notice that this valuation formula is recursive since the funding and hedging amounts in the RHS of (5) depend on future values of the hedge. This structure also breaks the linearity of adjustments, as it is a nonlinear valuation formula, in general. This nonlinear valuation formula is intuitively appealing because it specifies the discount factors used for each term:

- $\quad\left(\bar{c}_{u}-r_{u}\right)$ for the cost-of-carry of the collateral account;

- $\left(r_{u}-\bar{f}_{u}\right)$ for the costs due to the funding account;

- $\quad\left(r_{u}-\bar{h}_{u}\right)$ for the costs due to hedging in the repo market;

where the effective funding rate $\bar{f}$ equals $\bar{f}_{t}:=f_{t}^{l} \mathbb{1}_{\left\{F_{t} \geq 0\right\}}+f_{t}^{b} \mathbb{1}_{\left\{F_{t}<0\right\}}$ and the effective repo rate $\bar{h}$ is given by $\bar{h}_{t}:=h_{t}^{l} \mathbb{1}_{\left\{F_{t}^{S} \geq 0\right\}}+h_{t}^{b} \mathbb{1}_{\left\{F_{t}^{S}<0\right\}}$.

The "adjusted price" of the contract in Proposition 1 is different form the "clean price" in (1) because it also accounts for credit risk, funding and collateral costs, which are the additional risk factors that gave rise in isolation to the valuation adjustments (or XVAs). The combined effect of these additional factors was illustrated numerically in [32] in the case of a vulnerable call option, via a sensitivity analysis that highlighted the impact of each such factor, as well as jointly in their interaction. For additional studies on counterparty risk see [40-42] in the case of longevity swaps, and [43] for considerations on the wrongway risk.

\subsection{Risk-Neutral Approach to Nonlinear Valuation Adjustments}

The risk-neutral price of the collateralized defaultable contract $(A, R, C, \tau)$ can be represented as in Proposition 1. We specialize the terms in the first line of (5) in order to highlight the valuation adjustments implicit in (5). For simplicity, we will henceforth assume that the event $\left\{\tau_{C}=\tau_{I}\right\}$ is negligible under $\mathbb{Q}^{r}$, so that (4) reduces to

$$
\vartheta_{\tau}(R, C)=Q_{\tau}-\mathbb{1}_{\left\{\tau=\tau_{C}\right\}} L_{C} Y^{+}+\mathbb{1}_{\left\{\tau=\tau_{I}\right\}} L_{I} Y^{-} .
$$

The expressions for the funding costs are given by

$$
\varphi_{f}(t, \widehat{\tau}):=\int_{t}^{\widehat{\tau}} D(t, u) F_{u}\left(r_{u}-\bar{f}_{u}\right) d u
$$

and for either the cost/benefit of carry of the repo transaction,

$$
\varphi_{h}(t, \widehat{\tau}):=\int_{t}^{\widehat{\tau}} D(t, u) F_{u}^{S}\left(r_{u}-\bar{h}_{u}\right) d u .
$$


If we distinguish between the borrowing and lending of cash from treasury explicitly, then we obtain

$$
\varphi_{f}(t, \widehat{\tau})=\int_{t}^{\widehat{\tau}} D(t, u)\left(F_{u}^{-}\left(f_{u}^{b}-r_{u}\right)-F_{u}^{+}\left(f_{u}^{l}-r_{u}\right)\right) d u=\varphi_{f^{b}}(t, \widehat{\tau})-\varphi_{f^{l}}(t, \widehat{\tau}) .
$$

Notice that if the investor can borrow and lend at risk-free rate so that $\bar{f}=r$, then $\varphi_{f}(t, \widehat{\tau})$ vanishes.

Similarly, for $\varphi_{h}(t, \widehat{\tau})$ we obtain

$$
\varphi_{h}(t, \widehat{\tau})=\int_{t}^{\widehat{\tau}} D(t, u)\left(\left(F_{u}^{S}\right)^{-}\left(h_{u}^{b}-r_{u}\right)-\left(F_{u}^{S}\right)^{+}\left(h_{u}^{l}-r_{u}\right)\right) d u=\varphi_{h^{b}}(t, \widehat{\tau})-\varphi_{h^{l}}(t, \widehat{\tau}) .
$$

When $f^{b} \geq f^{l} \geq r$ and $h^{b} \geq h^{l} \geq r$, the funding benefit adjustments are

$$
\operatorname{FBA}_{t}^{f}=\mathbb{E}_{\mathbb{Q}^{r}}\left(\varphi_{f^{l}}(t, \widehat{\tau}) \mid \mathcal{G}_{t}\right), \quad \mathrm{FBA}_{t}^{h}=\mathbb{E}_{\mathbb{Q}^{r}}\left(\varphi_{h^{l}}(t, \widehat{\tau}) \mid \mathcal{G}_{t}\right)
$$

and the funding cost adjustments are given by

$$
\mathrm{FCA}_{t}^{f}=\mathbb{E}_{\mathbb{Q}^{r}}\left(\varphi_{f^{b}}(t, \widehat{\tau}) \mid \mathcal{G}_{t}\right), \quad \mathrm{FCA}_{t}^{h}=\mathbb{E}_{\mathbb{Q}^{r}}\left(\varphi_{h^{b}}(t, \widehat{\tau}) \mid \mathcal{G}_{t}\right),
$$

leading to the funding valuation adjustment

$$
\mathrm{FVA}_{t}:=\mathrm{FCA}_{t}^{f}+\mathrm{FCA}_{t}^{h}-\left(\mathrm{FBA}_{t}^{f}+\mathrm{FBA}_{t}^{h}\right) .
$$

Recall that $Q$ is the CSA close-out valuation process of the contract inclusive of the

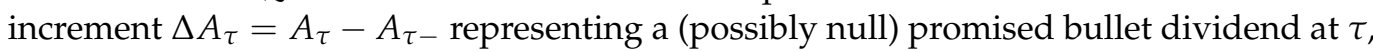
and $C_{\tau-}$ is the value of the collateral process $C$ at the moment of the first default. Then, we introduce the usual debit valuation adjustment

$$
\mathrm{DVA}_{t}=\mathbb{E}_{\mathbb{Q}^{r}}\left(\mathbb{1}_{\left\{\tau=\tau_{I} \leq T\right\}} D(t, \tau) L_{I}\left(Q_{\tau}-C_{\tau-}\right)^{-} \mid \mathcal{G}_{t}\right),
$$

the credit valuation adjustment

$$
\mathrm{CVA}_{t}=\mathbb{E}_{\mathbb{Q}^{r}}\left(\mathbb{1}_{\left\{\tau=\tau_{C} \leq T\right\}} D(t, \tau) L_{C}\left(Q_{\tau}-C_{\tau-}\right)^{+} \mid \mathcal{G}_{t}\right)
$$

and the collateral liquidity valuation adjustment

$$
\operatorname{LVA}_{t}:=\mathbb{E}_{\mathbb{Q}^{r}}\left(\gamma(t, \widehat{\tau}, C) \mid \mathcal{G}_{t}\right)
$$

It is now easy to check that the risk-neutral price for the collateralized defaultable contract $(A, R, C, \tau)$ can be represented in terms of XVAs as follows:

$$
\begin{aligned}
\pi_{t}^{r}(A, R, C, \tau) & =\pi_{t}^{r}(\widetilde{A})-\mathbb{E}_{\mathbb{Q}^{r}}\left(\mathbb{1}_{\{\tau \leq T\}} Q_{\tau} \mid \mathcal{G}_{t}\right)-\mathrm{DVA}_{t}+\mathrm{CVA}_{t}+\mathrm{FVA}_{t}+\mathrm{LVA}_{t} \\
& =\pi_{t}^{r}(A)-\mathrm{DVA}_{t}+\mathrm{CVA}_{t}+\mathrm{FVA}_{t}+\mathrm{LVA}_{t}
\end{aligned}
$$

where the last equality holds under the risk-free close-out convention. Note here that the XVA terms are not additive since the terms interact with one another. Furthermore, there are DVA2 terms and external funding benefits that have been treated in other works (see [3] for details).

\section{Valuation by Replication in Linear Multi-Curve Markets}

In the replication approach, the valuation of an OTC derivative focuses on computation of the price asked by the investor from their the counterparty in such a contract, under the assumption that it is based on investor's costs of replication through a self-financing trading strategy. Let us introduce the notation for interest rates and market prices of all traded assets in our generic market model. 
Repo markets. We denote by $\left(S^{1}, S^{2}, \ldots, S^{d}\right)$ the collection of prices of $d$ non-defaultable risky assets, which do not pay dividends. Let $B^{i, l}$ (respectively, $B^{i, b}$ ) stand for the lending (respectively, borrowing) repo account corresponding to the $i$ th risky non-defaultable asset. In the special case when $B^{i, l}=B^{i, b}$, the single repo account for the asset $S^{i}$ is denoted by $B^{i}$. We assume that $d B_{t}^{i, l}=h_{t}^{i, l} B_{t}^{i, l} d t, d B_{t}^{i, b}=h_{t}^{i, b} B_{t}^{i, b} d t$ and $d B_{t}^{i}=h_{t}^{i} B_{t}^{i} d t$ and the processes $S^{1}, S^{2}, \ldots, S^{d}$ are $\mathbb{G}$-semimartingales. Repo markets are nowadays well established for bonds and equities, although the lower quality as collateral means that the equity repo rate is invariably higher than investment-grade bond repo.

Unsecured trading. Let $\left(S^{d+1}, S^{d+2}, \ldots, S^{m}\right)$ be the collection of prices of $m$ nondefaultable risky assets, which do not pay dividends and are traded through unsecured funding from the bank's treasury. We assume that the processes $S^{d+1}, S^{d+2}, \ldots, S^{d+m}$ are $\mathbb{G}$-semimartingales. The lending (respectively, borrowing) cash account $B^{l}$ (respectively, $B^{b}$ ) can be used by the investor for unsecured lending (respectively, borrowing) of cash from the bank's treasury. When the borrowing and lending treasury rates coincide, the single treasury account is denoted by $B^{f}$. It is assumed that $d B_{t}^{l}=f_{t}^{l} B_{t}^{l} d t, d B_{t}^{b}=f_{t}^{b} B_{t}^{b} d t$ and $d B_{t}^{f}=f_{t} B_{t}^{f} d t$ where the treasury funding rates $f^{l}, f^{b}$ and $f$ are $\mathbb{G}$-adapted processes.

Defaultable securities. In order to guarantee that hedging of default risk is feasible, we also postulate that some defaultable securities are available for trade. Specifically, let $Z^{1}(t, T)$ and $Z^{2}(t, T)$ be the prices of $T$-maturity unit zero-coupon bonds issued by the investor's bank and the counterparty's entity. Of course, it is also possible to introduce credit default swaps (CDSs) in the present market model (see, e.g., Brigo et al. [32]). Let $\tau=\tau_{1} \wedge \tau_{2}=\tau_{I} \wedge \tau_{C}$ where $\tau_{1}=\tau_{I}$ and $\tau_{2}=\tau_{C}$ are $\mathbb{G}$-stopping times, representing the default times of the investor and the counterparty, respectively. As before, we denote by $\widehat{\tau}:=\tau \wedge T$ the effective maturity of the contract.

\subsection{Linear Markets with Funding Costs and Default Risk}

We start by examining a special case of the linear market with funding costs and defaults. By the linear market, we mean a particular instance of a general semimartingale model where the borrowing and lending rates for the funding account are equal, and the repo rates are also symmetric.

\subsubsection{Clean Price of a Financial Contract}

We first recall the definition of a self-financing trading strategy (also known as a dynamic portfolio) associated with a non-defaultable uncollateralized contract within the linear setup (see, for instance, Definition 2.3 in [24]). We henceforth assume that the investor decided to enter at time 0 into an OTC contract with the cumulative cash flows process $A$, which is specified by the clauses of the contract, and a (yet unknown) initial price $p_{0}$. Notice, however, that the contract is not assumed to be a traded asset after time 0 and thus we assume that the investor invests all their wealth in traded assets and funding accounts. Nevertheless, the dynamics of the value of his dynamic portfolio depend on the process $A$ and thus we use the notation $V_{t}^{p}(\varphi, A)$.

Definition 5. The value process of a portfolio $\varphi=\left(\psi^{f}, \kappa^{1}, \kappa^{2}, \psi^{1}, \ldots, \psi^{d}, \xi^{1}, \ldots, \xi^{m}\right)$ of traded assets and the corresponding funding accounts equals

$$
\begin{aligned}
V_{t}^{p}(\varphi, A) & :=\psi_{t}^{f} B_{t}^{f}+\sum_{j=1}^{2} \kappa_{t}^{j} Z^{j}(t, T)+\sum_{i=1}^{d}\left(\psi_{t}^{i} B_{t}^{i}+\xi_{t}^{i} S_{t}^{i}\right)+\sum_{i=d+1}^{m} \xi_{t}^{i} S_{t}^{i} \\
& =\psi_{t}^{f} B_{t}^{f}+\sum_{j=1}^{2} \kappa_{t}^{j} Z^{j}(t, T)+\sum_{i=d+1}^{m} \xi_{t}^{i} S_{t}^{i}=F_{t}+\sum_{j=1}^{2} Z_{t}^{j}+\sum_{i=d+1}^{m} H_{t}^{i}
\end{aligned}
$$

where we denote $F_{t}:=\psi_{t}^{f} B_{t}^{f}, Z_{t}^{j}:=\kappa_{t}^{j} Z^{j}(t, T)$ and $H_{t}^{i}:=\xi_{t}^{i} S_{t}^{i}$. We have also used the repo trading constraint $\psi_{t}^{i} B_{t}^{i}+\xi_{t}^{i} S_{t}^{i}=0$ for $i=1,2, \ldots, d$, which means that all long or short trades in the asset $S^{i}$ for $i=1,2, \ldots, d$ are funded using the account $B^{i}$ with the repo rate $h^{i}$. Put another way, 
it is postulated that $H_{t}^{i}=\xi_{t}^{i} S_{t}^{i}=-\psi_{t}^{i} B_{t}^{i}$ for $i=1,2, \ldots, d$. We say that a trading strategy $\varphi$ is self-financing inclusive of a contract $A$ if the value process $V^{p}(\varphi, A)$ satisfies (8) and

$$
\begin{aligned}
d V_{t}^{p}(\varphi, A) & =\psi_{t}^{f} d B_{t}^{f}+\sum_{j=1}^{2} \kappa_{t}^{j} d Z^{j}(t, T)+\sum_{i=1}^{d}\left(\psi_{t}^{i} d B_{t}^{i}+\xi_{t}^{i} d S_{t}^{i}\right)+\sum_{i=d+1}^{m} \xi_{t}^{i} d S_{t}^{i}+d A_{t} \\
& =\psi_{t}^{f} d B_{t}^{f}+\sum_{j=1}^{2} \kappa_{t}^{j} d Z^{j}(t, T)-\sum_{i=1}^{d} \xi_{t}^{i} S_{t}^{i}\left(B_{t}^{i}\right)^{-1} d B_{t}^{i}+\sum_{i=1}^{d} \xi_{t}^{i} d S_{t}^{i}+\sum_{i=d+1}^{m} \xi_{t}^{i} d S_{t}^{i}+d A_{t} .
\end{aligned}
$$

We assume that the pre-trading endowment of the investor is null. Hence, the initial price $p_{0}$ received by the investor after they entered into an uncollateralized contract $A$ is equal both to their initial wealth $V_{0}(\varphi, A)$ and the initial value $V_{0}^{p}(\varphi, A)$ of their portfolio. However, when a contract is collateralized, then the investor's initial wealth is still equal to $p_{0}$, but the initial value of the investor's portfolio equals $V_{0}^{p}(\varphi, A)=p_{0}+C_{0}$ where $C_{0}$ is the cash collateral, which is either pledged or received by the investor at time 0 . It is expected that $C_{0}>0$ if $p_{0}>0$, for instance, the seller of an option is posting collateral to the buyer. Our goal is to derive a probabilistic representation for the investor's unilateral initial price $p_{0}$ using replication-based arguments.

Since $\left(B_{t}^{i}\right)^{-1} d B_{t}^{i}=h_{t}^{i} d t$ for $i=1,2, \ldots, d$, we obtain from (9) the following:

$$
d V_{t}^{p}(\varphi, A)=f_{t} F_{t} d t+\sum_{j=1}^{2} \kappa_{t}^{j} d Z^{j}(t, T)+\sum_{i=1}^{d} \xi_{t}^{i}\left(d S_{t}^{i}-h_{t}^{i} S_{t}^{i} d t\right)+\sum_{i=d+1}^{m} \xi_{t}^{i} d S_{t}^{i}+d A_{t} .
$$

\subsubsection{Replication of a Non-Defaultable Collateralized Contract}

Let us now consider the case of a collateralized version of a contract $A$, which is denoted as $(A, C)$, where the margin account $C$ is assumed to be an exogenously given $\mathbb{G}$-semimartingale. We find it convenient to interpret the margin account as an additional stream of cash flows added to the process $A$, which was assumed to specify the clean (that is, uncollateralized and non-defaultable) version of the contract. For concreteness, we postulate that the cash collateral $C$ is rehypothecated, that is, it can be used for their trading purposes by collateral receiver. The process $C$ and its remuneration through accounts $B^{c, l}$ and $B^{c, b}$ (or, equivalently, the rates $c^{l}$ and $c^{b}$ ) are included in the definition of the process $A^{C}$, given by (11), and thus they directly affect the dynamics of portfolio's value, as specified by the self-financing condition of Definition 6. In the case of a non-defaultable collateralized contract with the margin process $C$ and collateral accrual rates $c^{l}$ and $c^{b}$ for the margin account, to compute the price and hedge for a collateralized contract, it suffices to replace in (9) the process $A$ by the process $A^{C}$ given by the following expression:

$$
\begin{aligned}
A_{t}^{C} & :=A_{t}+C_{t}+\int_{0}^{t} C_{u}^{-}\left(B_{u}^{c, l}\right)^{-1} d B_{u}^{c, l}-\int_{0}^{t} C_{u}^{+}\left(B_{u}^{c, b}\right)^{-1} d B_{u}^{c, b} \\
& =A_{t}+C_{t}+\int_{0}^{t}\left(c_{u}^{l} C_{u}^{-}-c_{u}^{b} C_{u}^{+}\right) d u=A_{t}+C_{t}-\int_{0}^{t} \bar{c}_{u} C_{u} d u
\end{aligned}
$$

where $C_{t}=C_{t}^{+}-C_{t}^{-}$for every $t \in[0, T]$ and where we use the effective collateral accrual rate, which equals

$$
\bar{c}_{t}:=c_{t}^{l} \mathbb{1}_{\left\{C_{t}<0\right\}}+c_{t}^{b} \mathbb{1}_{\left\{C_{t} \geq 0\right\}} .
$$

We thus see that the collateralized contract $(A, C)$ can be formally identified with the stream $A^{C}$ of cash flows. Note that the process $V^{p}\left(\varphi, A^{C}\right)$ is the value process of the investor's dynamic portfolio, whereas the process $V\left(\varphi, A^{C}\right):=V^{p}\left(\varphi, A^{C}\right)-C$ represents the investor's wealth In particular, their terminal wealth satisfies $V_{T}\left(\varphi, A^{\mathrm{C}}\right)=V_{T}^{p}\left(\varphi, A^{\mathrm{C}}\right)-$ $C_{T}$. We will now describe the dynamics of the value process of a self-financing trading strategy $\varphi$ inclusive of cash flows of a collateralized contract $(A, C)$. As was already mentioned, it suffices to extend Definition 5 to the case of a non-defaultable collateralized contract. 
Definition 6. We say that a trading strategy $\varphi$ is self-financing inclusive of a non-defaultable collateralized contract $(A, C)$ if the value process $V^{p}\left(\varphi, A^{C}\right)$ satisfies (8) and

$$
d V_{t}^{p}\left(\varphi, A^{C}\right)=f_{t} F_{t} d t+\sum_{j=1}^{2} \kappa_{t}^{j} d Z^{j}(t, T)+\sum_{i=1}^{d} \xi_{t}^{i}\left(d S_{t}^{i}-h_{t}^{i} S_{t}^{i} d t\right)+\sum_{i=d+1}^{m} \xi_{t}^{i} d S_{t}^{i}+d A_{t}^{C} .
$$

\subsubsection{Replication of a Defaultable Collateralized Contract}

In the next step, we introduce the concept of replication of a collateralized defaultable contract up its effective maturity date $\widehat{\tau}=\tau \wedge T$. To this end, we first define the stream of cash flows for a collateralized defaultable contract $(A, R, C, \tau)$. We set, for every $t \in[0, T]$,

$$
\widetilde{A}_{t}^{C}=\mathbb{1}_{\{t<\tau\}} A_{t}+\mathbb{1}_{\{t \geq \tau\}} A_{\tau-}+\mathbb{1}_{\{t<\tau\}} C_{t}+\mathbb{1}_{\{t \geq \tau\}} C_{\tau-}-\int_{0}^{t \wedge \tau} \bar{c}_{u} C_{u} d u
$$

and

$$
A_{t}^{R, C}=\widetilde{A}_{t}^{C}+\mathbb{1}_{\{t \geq \tau\}} R_{\tau} .
$$

The random variable $R_{\tau}$ represents a generic cash flow at time $\tau$ if the first default occurs either before or at $T$. Then the process $A^{R, C}$ gives all the cash flows of a defaultable collateralized contract $(A, R, C, \tau)$ up to its effective maturity $\widehat{\tau}$. Definition 6 can be extended in such a way that the process $A^{R, C}$ determines the value process $V^{p}\left(\varphi, A^{R, C}\right)$ on the stochastic interval $[0, \widehat{\tau}]$ and thus also the terminal value $V_{\tau}^{p}\left(\varphi, A^{R, C}\right)$ on the event $\{\tau \leq T\}$.

Definition 7. We say that a trading strategy $\varphi$ is self-financing, inclusive of a collateralized defaultable contract $(A, R, C, \tau)$, if the value process $V^{p}\left(\varphi, A^{R, C}\right)$ satisfies on $[0, \widehat{\tau}]$ equality (8) and

$$
d V_{t}^{p}\left(\varphi, A^{R, C}\right)=f_{t} F_{t} d t+\sum_{j=1}^{2} \kappa_{t}^{j} d Z^{j}(t, T)+\sum_{i=1}^{d} \xi_{t}^{i}\left(d S_{t}^{i}-h_{t}^{i} S_{t}^{i} d t\right)+\sum_{i=d+1}^{m} \xi_{t}^{i} d S_{t}^{i}+d A_{t}^{R, C} .
$$

It is worth noting that $A_{t}^{R, C}=\widetilde{A}_{t}^{C}=A_{t}^{C}$ on the event $\{t<\tau\}$, and thus the equalities

$$
V_{t}\left(\varphi, A^{R, C}\right)=V_{t}^{p}\left(\varphi, A^{R, C}\right)-C_{t}=V_{t}^{p}\left(\varphi, \widetilde{A}^{C}\right)-C_{t}=V_{t}^{p}\left(\varphi, A^{C}\right)-C_{t}
$$

hold on the event $\{t<\tau\}$ for every $t \in[0, T]$. However, the values of $V^{p}\left(\varphi, \widetilde{A}^{C}\right)$ and $V^{p}\left(\varphi, A^{C}\right)$ depend also on the cash flow $R_{\tau}$ through the initial price $p_{0}$ of the contract. These considerations lead to the following definition of the investor's wealth, in which we assume that they enter into a collateralized defaultable contract $(A, R, C, \tau)$ at the initial price $p_{0}$ and apply a self-financing strategy up to the contract's effective maturity $\widehat{\tau}$.

Definition 8 (Wealth process). The investor's wealth process $V\left(\varphi, A^{R, C}\right)$ equals, on the event $\{t<\tau\}$ for every $t \in[0, T]$,

$$
V_{t}\left(\varphi, A^{R, C}\right)=V_{t}^{p}\left(\varphi, A^{R, C}\right)-C_{t}=V_{t}^{p}\left(\varphi, \widetilde{A}^{C}\right)-C_{t}
$$

and on the event $\{\tau \leq T\}$,

$$
V_{\tau}\left(\varphi, A^{R, C}\right)=V_{\tau}^{p}\left(\varphi, A^{R, C}\right)=V_{\tau}^{p}\left(\varphi, \widetilde{A}^{C}\right)+R_{\tau} .
$$

Note that Definition 8 is consistent with the fact that any particular specification the cash flow $R_{\tau}$ must also encompass the collateral either pledged or received by the investor just before the time of the first default, that is, the quantity $C_{\tau-}$. Incidentally, we note that under strong contagion, the collateral can be inadequate to cover the loss triggered by the default, so that a sizeable credit effect can be present even with daily collateralization, 
see [44] for a discussion and the related gap risk. We are now in a position to introduce the concept of a replicating strategy for a collateralized defaultable contract.

Definition 9 (Replication). We say that a self-financing strategy $\varphi$ replicates a collateralized defaultable contract $(A, R, C, \tau)$ if $V_{\widehat{\tau}}\left(\varphi, A^{R, C}\right)=0$ or, equivalently, the following equality holds

$$
V_{\widehat{\tau}}^{p}\left(\varphi, \widetilde{A}^{C}\right)=C_{T} \mathbb{1}_{\{\tau>T\}}-R_{\tau} \mathbb{1}_{\{\tau \leq T\}} .
$$

Then the investor's unilateral ex-dividend price $\pi(A, R, C, \tau)$ for the collateralized defaultable contract $(A, R, C, \tau)$ is given by, on the event $\{t<\tau\}$ for all $t \in[0, T]$,

$$
\pi_{t}(A, R, C, \tau)=V_{t}\left(\varphi, A^{R, C}\right)=V_{t}^{p}\left(\varphi, \widetilde{A}^{C}\right)-C_{t} .
$$

Remark 1. It is clear that Definitions 8 and 9 cover also the valuation of non-defaultable contracts. Formally, it suffices to postulate that $\tau>T$. In that case, we have that $V_{t}\left(\varphi, A^{C}\right)=$ $V_{t}^{p}\left(\varphi, A^{C}\right)-C_{t}$ for every $t \in[0, T]$ and replication of a contract $(A, C)$ means that $V_{T}\left(\varphi, A^{C}\right)=0$ or, equivalently, that $V_{T}^{p}\left(\varphi, A^{C}\right)=C_{T}$.

\subsection{Valuation in a Linear Multi-Curve Market}

For the sake of clarity of presentation, we first examine the valuation of a nondefaultable collateralized contract and thus we now assume that defaultable bonds are not traded. Furthermore, to emphasize that the default times are not modeled, we denote the filtration by $\mathbb{F}=\left(\mathcal{F}_{t}\right)_{t \in[0, T]}$, rather than $\mathbb{G}=\left(\mathcal{G}_{t}\right)_{t \in[0, T]}$, since the latter filtration includes also the information about default times. In view of Definition 9 and Remark 1 , the replication of a collateralized contract $(A, C)$ by a self-financing trading strategy $\varphi$ means that $V_{T}\left(\varphi, A^{C}\right)=V_{T}^{p}\left(\varphi, A^{C}\right)-C_{T}=0$.

Definition 10 (Ex-dividend price). The investor's ex-dividend price $\pi(A, C)$ for the collateralized contract $(A, C)$ equals, for all $t \in[0, T]$,

$$
\pi_{t}(A, C)=V_{t}\left(\varphi, A^{C}\right)=V_{t}^{p}\left(\varphi, A^{C}\right)-C_{t}
$$

where $\varphi$ is a self-financing trading strategy that replicates $(A, C)$.

Assuming that a contract $(A, C)$ can be replicated, its initial investor's unilateral price $p_{0}$ is equal to $\pi_{0}(A, C)$. In the next result, we have that, for all $t \in[0, T]$,

$$
V_{t}^{p}\left(\varphi, A^{C}\right)=F_{t}+\sum_{i=d+1}^{m} H_{t}^{i}
$$

since we set $\kappa^{1}=\kappa^{2}=0$ in Equation (8). For concreteness, in the next result we assume that $A_{t}=\mathbb{1}_{\{t=T\}} X$ for all $t \in[0, T]$.

Lemma 1. Assume that bonds $Z^{1}$ and $Z^{2}$ are not traded and $A_{t}=\mathbb{1}_{\{t=T\}} X$, where $X$ is a square-integrable $\mathcal{F}_{T}$-measurable random variable. Then, the self-financing condition (13) yields the following dynamics for the investor's wealth process $V\left(\varphi, A^{C}\right)$ :

$$
d V_{t}\left(\varphi, A^{C}\right)=f_{t}\left(V_{t}\left(\varphi, A^{C}\right)-\sum_{i=d+1}^{m} H_{t}^{i}\right) d t+\sum_{i=1}^{d} \xi_{t}^{i}\left(d S_{t}^{i}-h_{t}^{i} S_{t}^{i} d t\right)+\sum_{i=d+1}^{m} \xi_{t}^{i} d S_{t}^{i}+d \widehat{C}_{t}
$$

where the process $\widehat{C}$ is given by

$$
\widehat{C}_{t}=\int_{0}^{t}\left(c_{u}^{l}-f_{u}\right) C_{u}^{-} d u-\int_{0}^{t}\left(c_{u}^{b}-f_{u}\right) C_{u}^{+} d u=\int_{0}^{t}\left(f_{u}-\bar{c}_{u}\right) C_{u} d u
$$


Proof. Equality (19) follows from (11), (13) and the equality $V\left(\varphi, A^{C}\right)=V^{p}\left(\varphi, A^{C}\right)-C$.

Example 2. Equation (19) can be interpreted as a backward stochastic differential Equation (BSDE) for $(Y, \xi)$, where $Y_{t}=\pi_{t}(A, C)$ for $t \in[0, T)$. For concreteness, let us examine the valuation of $a$ call option on the asset $S=S^{1}$. If the investor sells the call option, then $\Delta A_{T}^{S}=-\left(S_{T}-K\right)^{+}$and thus the terminal condition in the pricing BSDE is $Y_{T}^{S}=\left(S_{T}-K\right)^{+}$. This is, of course, consistent with the standard concept of replication of the call payoff by its writer. If the investor buys the call at time 0 , then $\Delta A_{T}^{b}=\left(S_{T}-K\right)^{+}$and thus the terminal condition becomes $Y_{T}^{b}=-\left(S_{T}-K\right)^{+}$, since to hedge their exposure, the buyer needs to replicate the payoff $-\left(S_{T}-K\right)^{+}$. Note that the collateral $C_{t}^{s}=-\left(C_{t}^{s}\right)^{-}$will be pledged at time $t$ by the investor who writes the call, but the collateral will be received by them if he buys the call, meaning that $C_{t}^{b}=\left(C_{t}^{b}\right)^{+}$in the latter case. In view of the symmetry of collateralization, it is natural to postulate that $C_{t}^{b}=-C_{t}^{s}$ for all $t \in[0, T]$, especially in the case where a collateral amount is exogenously determined. If $c^{l} \neq c^{b}$, then it is expected that

$$
\pi_{t}\left(A^{s}, C^{s}\right) \neq-\pi_{t}\left(-A^{s},-C^{s}\right)=-\pi_{t}\left(A^{b}, C^{b}\right)
$$

that is, the absolute value of the investor's price will depend on whether he is willing to sell or buy an option. In contrast, the equalities

$$
\pi_{t}\left(A^{s}, C^{s}\right)=-\pi_{t}\left(-A^{s},-C^{s}\right)=-\pi_{t}\left(A^{b}, C^{b}\right)
$$

are valid when $c^{l}=c^{b}=c$, even if $c \neq f$. This illustrates the general property that the buying/selling investor's prices are equal in the linear setup when $c^{l}=c^{b}=c$ if the symmetry of collateral is postulated. Formally, if $c^{l}=c^{b}=c$, then $\pi_{t}(A, C)=-\pi_{t}(-A,-C)$ for all $t \in[0, T]$ and an arbitrary specification of the processes $A$ and $C$.

\subsubsection{Auxiliary Lemma}

To obtain a general version of probabilistic representation for ex-dividend price, we will need to establish Lemma 2. We start by introducing the following notation:

$$
B_{t}^{\zeta^{j}}:=\exp \left(\int_{0}^{t} \zeta_{u}^{j} d u\right), B_{t}^{\gamma^{i}}:=\exp \left(\int_{0}^{t} \gamma_{u}^{i} d u\right), B_{t}^{v^{i}}:=\exp \left(\int_{0}^{t} v_{u}^{i} d u\right)
$$

where $\zeta^{j}, \gamma^{i}$, and $v^{i}$ are arbitrary $\mathbb{G}$-adapted and integrable processes. Then, the processes $\bar{Z}^{j}(t, T):=\left(B_{t}^{\zeta^{j}}\right)^{-1} Z^{j}(t, T)$ for $j=1,2$ satisfy

$$
d \bar{Z}^{j}(t, T)=\left(B_{t}^{\zeta^{j}}\right)^{-1}\left(d Z^{j}(t, T)-\zeta_{t}^{j} Z^{j}(t, T) d t\right) .
$$

For the risky assets traded under repo convention, we define the processes $\bar{S}^{i}:=$ $\left(B^{\gamma^{i}}\right)^{-1} S^{i}$ for $i=1,2, \ldots, d$, so that we can write

$$
d \bar{S}_{t}^{i}=\left(B_{t}^{\gamma^{i}}\right)^{-1}\left(d S_{t}^{i}-\gamma_{t}^{i} S_{t}^{i} d t\right), \quad i=1,2, \ldots, d
$$

Similarly, for the risky assets directly traded on the spot market, we set $\bar{S}^{i}:=\left(B^{v^{i}}\right)^{-1} S^{i}$ for $i=d+1, d+2, \ldots, m$ and thus

$$
d \bar{S}_{t}^{i}=\left(B_{t}^{v^{i}}\right)^{-1}\left(d S_{t}^{i}-v_{t}^{i} S_{t}^{i} d t\right), \quad i=d+1, d+2, \ldots, m .
$$

Definition 11. Let $(\zeta, \gamma, v)=\left(\zeta^{1}, \zeta^{2}, \gamma^{1}, \gamma^{2}, \ldots, \gamma^{d}, v^{d+1}, v^{d+2}, \ldots, v^{m}\right)$ be an $(m+2)$ dimensional, $\mathbb{G}$-adapted, integrable process. Then, we denote by $\mathbb{Q}^{\zeta, \gamma, v}$ a probability measure on $\left(\Omega, \mathcal{G}_{T}\right)$, such that the processes $\bar{Z}^{j}(t, T), j=1,2$ and $\bar{S}^{i}, i=1,2, \ldots, m$ are $\mathbb{Q}^{\zeta, \gamma, v}$-local martingales.

The existence of a probability measure $\mathbb{Q}^{\zeta, \gamma, \nu}$ is not obvious a priori, but it can be established in most market models encountered in the existing literature and thus we 
will henceforth postulate that such a probability measure is well defined. Then, from Definition 11, it follows that the processes

$$
Z^{j}(t, T)-\int_{0}^{t} \zeta_{u}^{j} Z^{j}(u, T) d u, \quad S_{t}^{i}-\int_{0}^{t} \gamma_{u}^{i} S_{u}^{i} d u, \quad S_{t}^{i}-\int_{0}^{t} v_{u}^{i} S_{u}^{i} d u
$$

are $\left(\mathbb{Q}^{\zeta, \gamma, v}, \mathbb{G}\right)$-local martingales. In other words, $\mathbb{Q}^{\zeta, \gamma, v}$ is a local martingale measure for prices $Z^{j}(t, T)$ discounted by $B^{\zeta^{j}}, S^{i}$ discounted with the processes $B^{\gamma^{i}}$ for $i=1,2, \ldots, d$ and $S^{i}$ discounted with the processes $B^{v^{i}}$ for $i=d+1, d+2, \ldots, m$.

For an arbitrary $\mathbb{G}$-adapted and integrable process $\eta$, we define the process $B^{\eta}$ by setting, for every $t \in[0, T]$,

$$
B_{t}^{\eta}:=\exp \left(\int_{0}^{t} \eta_{u} d u\right)
$$

and, for brevity, we write $D^{\eta}(t, s)=B_{t}^{\eta}\left(B_{s}^{\eta}\right)^{-1}$. The following lemma underpins the probabilistic approach to the valuation of contracts under funding costs.

Lemma 2. Assume that $V^{p}\left(\varphi, \widetilde{A}^{C}\right)$ is the value process of a self-financing trading strategy, in the sense of Definition 6, so that (13) holds for $t \in[0, T]$ with $A^{C}=\widetilde{A}^{C}$. Let $\eta$ be an arbitrary $\mathbb{G}$-adapted and integrable process and let the process $V^{\eta}\left(\varphi, \widetilde{A}^{C}\right)$ be given by

$$
\begin{aligned}
V_{t}^{\eta}\left(\varphi, \widetilde{A}^{C}\right):= & V_{t}^{p}\left(\varphi, \widetilde{A}^{C}\right)+\int_{0}^{t} D^{\eta}(t, u) \alpha_{u} F_{u} d u+\sum_{j=1}^{2} \int_{0}^{t} D^{\eta}(t, u) \delta_{u}^{j} Z_{u}^{j} d u \\
& +\sum_{i=1}^{d} \int_{0}^{t} D^{\eta}(t, u) \beta_{u}^{i} H_{u}^{i} d u+\sum_{i=d+1}^{m} \int_{0}^{t} D^{\eta}(t, u) \chi_{u}^{i} H_{u}^{i} d u-\int_{(0, t]} D^{\eta}(t, u) d \widetilde{A}_{u}^{C} .
\end{aligned}
$$

If the following equalities hold for every $t \in[0, T]$

$$
\alpha_{t}=\eta_{t}-f_{t}, \quad \delta_{t}^{j}=\eta_{t}-\zeta_{t}^{j}, \quad \beta_{t}^{i}=h_{t}^{i}-\gamma_{t}^{i}, \quad \chi_{t}^{i}=\eta_{t}-v_{t}^{i},
$$

then the process $\bar{V}^{\eta}\left(\varphi, \widetilde{A}^{C}\right):=\left(B^{\eta}\right)^{-1} V^{\eta}\left(\varphi, \widetilde{A}^{C}\right)$ is a $\left(\mathbb{Q}^{\zeta, \gamma, v}, \mathbb{G}\right)$-local martingale.

Proof. Let us denote $V^{\eta}=V^{\eta}\left(\varphi, \widetilde{A}^{C}\right)$ and $V^{p}=V^{p}\left(\varphi, \widetilde{A}^{C}\right)$. From Equation (21), we obtain

$$
d V_{t}^{\eta}=d V_{t}^{p}+\alpha_{t} F_{t} d t+\sum_{j=1}^{2} \delta_{t}^{j} Z_{t}^{j} d t+\sum_{i=1}^{d} \beta_{t}^{i} H_{t}^{i} d t+\sum_{i=d+1}^{m} \chi_{t}^{i} H_{t}^{i} d t+\left(V_{t}^{\eta}-V_{t}^{p}\right) \eta_{t} d t-d \widetilde{A}_{t}^{C} .
$$

Since $V_{t}^{p}=F_{t}+\sum_{j=1}^{2} Z_{t}^{j}+\sum_{i=d+1}^{m} H_{t}^{i}$, we obtain

$$
\begin{aligned}
& d V_{t}^{\eta}-\eta_{t} V_{t}^{\eta} d t=f_{t} F_{t} d t+\sum_{j=1}^{2} \kappa_{t}^{j} d Z^{j}(t, T)+\sum_{i=1}^{d} \xi_{t}^{i}\left(d S_{t}^{i}-h_{t}^{i} S_{t}^{i} d t\right)+\sum_{i=d+1}^{m} \xi_{t}^{i} d S_{t}^{i}+\alpha_{t} F_{t} d t \\
& +\sum_{j=1}^{2} \delta_{t}^{j} Z_{t}^{j} d t+\sum_{i=1}^{d} \beta_{t}^{i} H_{t}^{i} d t+\sum_{i=d+1}^{m} \chi_{t}^{i} H_{t}^{i} d t-\eta_{t} V_{t}^{p} d t
\end{aligned}
$$

which in turn yields 


$$
\begin{aligned}
d V_{t}^{\eta}-\eta_{t} V_{t}^{\eta} d t & =\left(\alpha_{t}+f_{t}-\eta_{t}\right) F_{t} d t+\sum_{j=1}^{2}\left(\delta_{t}^{j}+\zeta_{t}^{j}-\eta_{t}\right) Z_{t}^{j} d t+\sum_{j=1}^{2} \kappa_{t}^{j}\left(d Z^{j}(t, T)-\zeta_{t}^{j} Z^{j}(t, T) d t\right) \\
& +\sum_{i=1}^{d}\left(\beta_{t}^{i}+\gamma_{t}^{i}-h_{t}^{i}\right) H_{t}^{i} d t+\sum_{i=1}^{d} \xi_{t}^{i}\left(d S_{t}^{i}-\gamma_{t}^{i} S_{t}^{i} d t\right)+\sum_{i=d+1}^{m}\left(\chi_{t}^{i}+v_{t}^{i}-\eta_{t}\right) H_{t}^{i} d t \\
& +\sum_{i=d+1}^{m} \xi_{t}^{i}\left(d S_{t}^{i}-v_{t}^{i} S_{t}^{i} d t\right)
\end{aligned}
$$

Since $d \bar{V}_{t}^{\eta}=\left(B_{t}^{\eta}\right)^{-1}\left(d V_{t}^{\eta}-\eta_{t} V_{t}^{\eta} d t\right)$, it is now clear that if the processes $\alpha, \delta, \beta$ and $\chi$ satisfy (22), then the process $\bar{V}^{\eta}=\left(B^{\eta}\right)^{-1} V^{\eta}$ is a $\left(\mathbb{Q}^{\zeta, \gamma, \nu}, \mathbb{G}\right)$-local martingale.

\subsubsection{Linear Probabilistic Valuation Formula}

We are in a position to prove the main result in this section, which gives a general probabilistic representation for the ex-dividend price. We stress that a financial interpretation of processes $\eta, \zeta^{1}, \zeta^{2}, \gamma^{i}$ for $i=1,2, \ldots, d$, and $v^{i}$ for $i=d+1, d+2, \ldots, m$ is completely irrelevant for the derivation of representations (24) and (25). Recall that we denote

$$
\widetilde{A}_{t}=\mathbb{1}_{\{t<\tau\}} A_{t}+\mathbb{1}_{\{t \geq \tau\}} A_{\tau-}, \quad \widetilde{C}_{t}=\mathbb{1}_{\{t<\tau\}} C_{t}+\mathbb{1}_{\{t \geq \tau\}} C_{\tau-},
$$

the effective collateral rate $\bar{c}$ is given by (12), and $\vartheta_{\tau}(R, C)=R_{\tau}+C_{\tau-}$ is the close-out payoff at $\tau$.

Theorem 1. Assume that the collateralized defaultable contract $(A, R, C, \tau)$ can be replicated by a trading strategy $\varphi$. If the associated process $\bar{V}^{\eta}\left(\varphi, \widetilde{A}^{C}\right)=\left(B^{\eta}\right)^{-1} V^{\eta}\left(\varphi, \widetilde{A}^{C}\right)$, where $V^{\eta}\left(\varphi, \widetilde{A}^{C}\right)$ is given by $(21)$, is a true $\left(\mathbb{Q}^{\zeta, \gamma, \nu}, \mathbb{G}\right)$-martingale, then the value process $V_{t}^{p}\left(\varphi, A^{R, C}\right)$ equals, on the event $\{t<\tau\}$ for every $t \in[0, T]$,

$$
\begin{aligned}
V_{t}^{p}\left(\varphi, A^{R, C}\right) & =\mathbb{E}_{\mathbb{Q} \zeta, \gamma, v}\left(-\int_{] t, \widehat{\tau}]} D^{\eta}(t, u) d \widetilde{A}_{u}^{C}+D^{\eta}(t, T) C_{T} \mathbb{1}_{\{\tau>T\}}-D^{\eta}(t, \tau) R_{\tau} \mathbb{1}_{\{\tau \leq T\}} \mid \mathcal{G}_{t}\right) \\
& +\mathbb{E}_{\mathbb{Q} \zeta, \gamma, v}\left(\int_{t}^{\widehat{\tau}} D^{\eta}(t, u)\left(\eta_{u}-f_{u}\right) F_{u} d u+\sum_{j=1}^{2} \int_{t}^{\widehat{\tau}} D^{\eta}(t, u)\left(\eta_{u}-\zeta_{u}^{j}\right) Z_{u}^{j} d u \mid \mathcal{G}_{t}\right) \\
& +\mathbb{E}_{\mathbb{Q} \zeta, \gamma, v}\left(\sum_{i=1}^{d} \int_{t}^{\widehat{\tau}} D^{\eta}(t, u)\left(h_{u}^{i}-\gamma_{u}^{i}\right) H_{u}^{i} d u+\sum_{i=d+1}^{m} \int_{t}^{\widehat{\tau}} D^{\eta}(t, u)\left(\eta_{u}-v_{u}^{i}\right) H_{u}^{i} d u \mid \mathcal{G}_{t}\right) .
\end{aligned}
$$

Furthermore, the ex-dividend price for the collateralized defaultable contract $(A, R, C, \tau)$ is given by the following probabilistic expression, on the event $\{t<\tau\}$ for every $t \in[0, T]$,

$$
\begin{aligned}
& \pi_{t}(A, R, C, \tau)=\mathbb{E}_{\mathbb{Q}^{\zeta}, \gamma, v}\left(-\int_{] t, \widehat{\tau}]} D^{\eta}(t, u) d \widetilde{A}_{u}-D^{\eta}(t, \tau) \vartheta_{\tau}(R, C) \mathbb{1}_{\{\tau \leq T\}} \mid \mathcal{G}_{t}\right) \\
& +\mathbb{E}_{\mathbb{Q}^{\zeta, \gamma, v}}\left(\int_{t}^{\widehat{\tau}} D^{\eta}(t, u)\left(\eta_{u}-f_{u}\right) F_{u} d u+\sum_{j=1}^{2} \int_{t}^{\widehat{\tau}} D^{\eta}(t, u)\left(\eta_{u}-\zeta_{u}^{j}\right) Z_{u}^{j} d u \mid \mathcal{G}_{t}\right) \\
& +\mathbb{E}_{\mathbb{Q}^{\zeta, \gamma, v}}\left(\sum_{i=1}^{d} \int_{t}^{\widehat{\tau}} D^{\eta}(t, u)\left(h_{u}^{i}-\gamma_{u}^{i}\right) H_{u}^{i} d u+\sum_{i=d+1}^{m} \int_{t}^{\widehat{\tau}} D^{\eta}(t, u)\left(\eta_{u}-v_{u}^{i}\right) H_{u}^{i} d u \mid \mathcal{G}_{t}\right) \\
& +\mathbb{E}_{\mathbb{Q}^{\zeta, \gamma, v}}\left(\int_{t}^{\widehat{\tau}} D^{\eta}(t, u)\left(\bar{c}_{u}-\eta_{u}\right) C_{u} d u \mid \mathcal{G}_{t}\right) .
\end{aligned}
$$

Proof. Let $\varphi$ be a trading strategy which replicates the contract $(A, R, C, \tau)$. In view of Definition 9 , this means that

$$
V_{\widehat{\tau}}^{p}\left(\varphi, \widetilde{A}^{C}\right)=C_{T} \mathbb{1}_{\{\tau>T\}}-R_{\tau} \mathbb{1}_{\{\tau \leq T\}} .
$$


Equation (24) is thus an immediate consequence of the martingale property of $\bar{V}^{\eta}\left(\varphi, \widetilde{A}^{C}\right)$, the fact that $\widehat{\tau}$ is a $\mathbb{G}$-stopping time and equality (26). It remains to show that (25) can be deduced from (24) and the definition of the ex-dividend price. On the event $\{t<\tau\}$, we have that $\pi_{t}(A, R, C, \tau)=V_{t}\left(\varphi, A^{R, C}\right)=V_{t}^{p}\left(\varphi, \widetilde{A}^{C}\right)-C_{t}$ (see (18)). Note that (14) gives $d \widetilde{A}_{t}^{C}=d \widetilde{A}_{t}+d \widetilde{C}_{t}-\bar{c}_{t} C_{t} d t$. The integration by parts formula yields, on the event $\{\tau>T\}$,

$$
\begin{aligned}
\int_{] t, \widehat{\tau}]}\left(B_{u}^{\eta}\right)^{-1} d \widetilde{C}_{u} & =\int_{] t, T]}\left(B_{u}^{\eta}\right)^{-1} d C_{u}=C_{T}\left(B_{T}^{\eta}\right)^{-1}-C_{t}\left(B_{t}^{\eta}\right)^{-1}-\int_{t}^{T} C_{u} d\left(B_{u}^{\eta}\right)^{-1} \\
& =C_{T}\left(B_{T}^{\eta}\right)^{-1}-C_{t}\left(B_{t}^{\eta}\right)^{-1}+\int_{t}^{T} \eta_{u}\left(B_{u}^{\eta}\right)^{-1} C_{u} d u
\end{aligned}
$$

whereas on the event $\{\tau \leq T\}$, we obtain

$$
\begin{aligned}
\int_{] t, \widehat{\tau}]}\left(B_{u}^{\eta}\right)^{-1} d \widetilde{C}_{u} & =\int_{] t, \tau]}\left(B_{u}^{\eta}\right)^{-1} d \widetilde{C}_{u}=C_{\tau-}\left(B_{\tau}^{\eta}\right)^{-1}-C_{t}\left(B_{t}^{\eta}\right)^{-1}-\int_{t}^{\tau} C_{u} d\left(B_{u}^{\eta}\right)^{-1} \\
& =C_{\tau-}\left(B_{\tau}^{\eta}\right)^{-1}-C_{t}\left(B_{t}^{\eta}\right)^{-1}+\int_{t}^{\tau} \eta_{u}\left(B_{u}^{\eta}\right)^{-1} C_{u} d u .
\end{aligned}
$$

It is now easy to check that (25) is a direct consequence of (24).

The master Equation (25) is henceforth referred to as the linear valuation formula since in order to identify the processes $F, Z^{j}$ and $H^{i}$ it suffices to solve a linear BSDE for which a solution is explicitly known in a typical financial model. This property should be contrasted with the nonlinear valuation formula of Theorem 2, where the coefficients involve the effective rates $\bar{f}, \overline{h^{i}}$, and $\bar{c}$, which are given by (38), (39), and (12), respectively, and hence they are nonlinear functions of unknown processes $F, Z^{j}$ and $H^{i}$. Therefore, in order to compute the price using (41), one needs solve a nonlinear BSDE (we refer to Section 4.3 for a very brief discussion of BSDEs in the present context).

We stress that Equation (25) and some of its consequences (see (28) and (34)) essentially differ from the classical risk-neutral valuation formula, which relies on computation of conditional expectation of discounted future payoffs under the risk-neutral probability. Since the processes $F, Z^{j}$ and $H^{i}$ appear in the right-hand side of (25), (28), and (34), it is not possible to compute the contract's price from these formulae without finding also its replicating strategy. The latter feature should be contrasted with the pricing Equation (27), where indeed the price is given by a simple conditional expectation of discounted future payoffs under a suitable probability measure, and thus (27) can be seen as a direct extension of the classical risk-neutral pricing formula. It is worth recalling that the link between a solution to the linear BSDE and the risk-neutral pricing formula was established in seminal papers by El Karoui et al. [45], El Karoui and Quenez [46], which constitute a basis for the present research. In essence, they show that if a pair $(Y, Z)$ is a solution to a linear BSDE under the real-world probability, then the process $Y$ can be found by taking the conditional expectation of properly discounted terminal condition under an equivalent risk-neutral probability. All our results in Section 3 can be seen as far-reaching extensions of that conclusion to multi-curve markets with credit risk.

We will now consider some applications of Theorem 1 . The first corollary furnishes a natural probabilistic representation for the ex-dividend price of a collateralized defaultable contract $(A, R, C, \tau)$. It should be noted that we obtain here a closed-form solution to the valuation problem and the right-hand side in (27) can be computed explicitly for several cases of interest. We denote $D^{f}(t, u):=B_{t}^{f}\left(B_{u}^{f}\right)^{-1}$. 
Corollary 1. If $\eta=f, \zeta^{j}=f, \gamma^{i}=h^{i}$ for $i=1,2, \ldots, d$ and $v^{i}=f$ for $i=d+1, d+2, \ldots, m$, then (24) yields the following expression for the ex-dividend price of a collateralized defaultable contract $(A, R, C, \tau)$ :

$$
\begin{aligned}
\pi_{t}(A, R, C, \tau) & =\mathbb{E}_{\mathbb{Q}^{f, h, f}}\left(-\int_{] t, T]} D^{f}(t, u) d \widetilde{A}_{u}-\vartheta_{\tau}(R, C) D^{f}(t, \tau) \mathbb{1}_{\{\tau \leq T\}} \mid \mathcal{G}_{t}\right) \\
& +\mathbb{E}_{\mathbb{Q}^{f, h, f}}\left(\int_{t}^{\widehat{\tau}} D^{f}(t, u)\left(\bar{c}_{u}-f_{u}\right) C_{u} d u \mid \mathcal{G}_{t}\right) .
\end{aligned}
$$

Corollary 1 is suitable when we are concerned with computation of the investor's unilateral price for $(A, R, C, \tau)$. In contrast, it does not offer an immediate decomposition of the all-inclusive price $\pi_{t}(A, R, C, \tau)$ in terms of its clean price and various valuation adjustments, which are of practical interest so that they were extensively studied in the existing financial literature. For this reason, we will present in the next section another application of Theorem 1, where the valuation adjustments, such as CVA, DVA, FVA, LVA, etc., will show explicitly. As mentioned in the introduction, the clean price is computed and used by the trading desk, whereas various valuation adjustments (i.e., XVAs) are handled by other divisions of a financial institution. Hence there is a clear need to obtain explicit decompositions of the all-inclusive price (sometimes called the exit price by practitioners) and this will be carried out in the foregoing subsections (see Corollary 2, Proposition 2, and Equation (35)).

\subsubsection{Linear Risk-Neutral Valuation with Funding, Defaults, and Collateralization}

Our next goal is to show how to obtain from Theorem 1 a version of the risk-neutral valuation formula with adjusted cash flows, which was first derived through different means in Pallavicini et al. [23] and Brigo et al. [2]. Recall that $r$ is a $\mathbb{G}$-adapted, integrable process and $B^{r}$ stands for the associated risk-free cash account

$$
B_{t}^{r}:=\exp \left(\int_{0}^{t} r_{u} d u\right)
$$

As before, we do not assume that the risk-free cash account $B^{r}$ is available to the investor and thus the risk-free rate process $r$ can be seen as a purely instrumental variable.

Upon setting $\eta=\zeta^{j}=\gamma^{i}=v^{i}=r$ in (25), we obtain representation (28), which is a version of the risk-neutral valuation with adjusted cash flows. To alleviate the notation, we henceforth write $\mathbb{Q}^{r}=\mathbb{Q}^{r, r, r}$. Furthermore, for the sake of symmetry, we write $F_{t}^{i}=\psi_{t}^{i} B_{t}^{i}=$ $-H_{t}^{i}$. Recall that $D(t, u):=B_{t}^{r}\left(B_{u}^{r}\right)^{-1}$.

Corollary 2. The ex-dividend price for the collateralized defaultable contract $(A, R, C, \tau)$ is given $b y$, on the event $\{t<\tau\}$ for every $t \in[0, T]$,

$$
\begin{aligned}
& \pi_{t}(A, R, C, \tau)=\mathbb{E}_{\mathbb{Q}^{r}}\left(-\int_{] t, \hat{\tau}]} D(t, u) d \widetilde{A}_{u}-D(t, \tau) \vartheta_{\tau}(R, C) \mathbb{1}_{\{\tau \leq T\}} \mid \mathcal{G}_{t}\right) \\
& +\mathbb{E}_{\mathbb{Q}^{r}}\left(\int_{t}^{\widehat{\tau}} D(t, u)\left(r_{u}-f_{u}\right) F_{u} d u+\sum_{i=1}^{d} \int_{t}^{\widehat{\tau}} D(t, u)\left(r_{u}-h_{u}^{i}\right) F_{u}^{i} d u \mid \mathcal{G}_{t}\right) \\
& +\mathbb{E}_{\mathbb{Q}^{r}}\left(\int_{t}^{\widehat{\tau}} D(t, u)\left(\bar{c}_{u}-r_{u}\right) C_{u} d u \mid \mathcal{G}_{t}\right) .
\end{aligned}
$$

Observe that, by an application of Definition 11 , the processes $\bar{Z}^{j}(t, T)=\left(B_{t}^{r}\right)^{-1} Z^{j}(t, T)$ for $j=1,2$ and $\bar{S}^{i}=\left(B_{t}^{r}\right)^{-1} S^{i}$ for $i=1,2, \ldots, m$ or, equivalently, the processes

$$
Z^{j}(t, T)-\int_{0}^{t} r_{u} Z^{j}(u, T) d u, \quad S_{t}^{i}-\int_{0}^{t} r_{u} S_{u}^{i} d u,
$$


are $\left(\mathbb{Q}^{r}, \mathbb{G}\right)$-local martingales and thus the probability measure $\mathbb{Q}^{r}$ can be interpreted as a classical risk-neutral probability, which is used to compute clean prices of financial derivatives.

Let us consider some special cases of Formula (28). Suppose that the bank's treasury rate $f$, the repo rates $h^{i}$, and the collateral accrual rates are all equal to the risk-free rate $r$. Then, Corollary 2 yields the following variant of the risk-neutral valuation formula for a collateralized defaultable contract

$$
\pi_{t}(A, R, C, \tau)=\mathbb{E}_{\mathbb{Q}^{r}}\left(-\int_{] t, \widehat{\tau}]} D(t, u) d \widetilde{A}_{u}-D(t, \tau) \vartheta_{\tau}(R, C) \mathbb{1}_{\{\tau \leq T\}} \mid \mathcal{G}_{t}\right) .
$$

If, in addition, the equality $R_{\tau}=-C_{\tau-}$ holds-meaning that the collateral is simply returned at the moment of the first default to the pledging party and no additional payoff occurs at time $\tau$ on the event $\{\tau \leq T\}$ 一then (29) further reduces to the following:

$$
\begin{aligned}
\pi_{t}(A, R, C, \tau) & =\mathbb{E}_{\mathbb{Q}^{r}}\left(-\int_{] t, \widehat{\tau}]} D(t, u) d \widetilde{A}_{u} \mid \mathcal{G}_{t}\right) \\
& =\mathbb{E}_{\mathbb{Q}^{r}}\left(-\mathbb{1}_{\{\tau>T\}} \int_{] t, T]} D(t, u) d A_{u}-\mathbb{1}_{\{\tau \leq T\}} \int_{] t, \tau)} D(t, u) d A_{u} \mid \mathcal{G}_{t}\right) .
\end{aligned}
$$

\subsection{Linear Valuation with XVAs under Risk-Free Close-Out}

Let us examine the consequences of the counterparty credit risk on the price of collateralized defaultable contract. To this end, we need to specify the CSA close-out valuation process $Q$ for the contract $(A, R, C, \tau)$. Let us stress that any specification for the process $Q$ should also include the promised payoff $A_{\tau}-A_{\tau-}$ at the moment of the first default. For instance, one could set (see (27))

$$
Q_{t}=\mathbb{E}_{\mathbb{Q}^{f, h, f}}\left(\int_{[t, T]} D^{f}(t, u) d A_{u} \mid \mathcal{G}_{t}\right)
$$

which would mean that the idiosyncratic funding costs of the investor would affect the CSA valuation. In the above equation, we use the same notation of Equation (27). In particular, we recall that the expectation is taken under the measure $\mathbb{Q}^{\zeta, \gamma, v}$, given in Definition 11, with the choice $\zeta=v=f$ and $\gamma=h$, and $D^{f}(t, u):=B_{t}^{f}\left(B_{u}^{f}\right)^{-1}$.

A more conventional form of the CSA close-out value hinges on the postulate that funding of all assets can be carried out at the risk-free rate $r$, and we henceforth follow this market convention. To be more specific, we set $f=h^{i}=r$ for $i=1,2, \ldots, d$ and $\tau>T$ in (28) in order to obtain Equality (31). This means that the close-out valuation $Q$ is given by the clean price of the contract.

Definition 12. The risk-free close-out valuation process $Q$ for the collateralized defaultable contract $(A, R, C, \tau)$ is given by

$$
Q_{t}:=\mathbb{E}_{\mathbb{Q}^{r}}\left(\int_{[t, T]} D(t, u) d A_{u} \mid \mathcal{G}_{t}\right)=-\pi_{t}^{r}(A)+\Delta A_{t}
$$

where $\Delta A_{t}=A_{t}-A_{t-}$ and $\pi_{t}^{r}(A), t \in[0, T]$ is the ex-dividend risk-free price of $A$, that is,

$$
\pi_{t}^{r}(A):=\mathbb{E}_{\mathbb{Q}^{r}}\left(-\int_{] t, T]} D(t, u) d A_{u} \mid \mathcal{G}_{t}\right)
$$

In view of Definition 12, at the moment of the first default we have, on the event $\{\tau \leq T\}$, that the close-out payoff that becomes due at time of default $\tau$ is as follows:

$$
Q_{\tau}=\mathbb{E}_{\mathbb{Q}^{r}}\left(\int_{[\tau, T]} D(t, \tau) d A_{u} \mid \mathcal{G}_{\tau}\right)=-\pi_{\tau}^{r}(A)+\Delta A_{\tau}
$$


Our goal is now to obtain the conventional valuation adjustments (XVAs). In this section, it is assumed that the recovery payoff $R_{\tau}$ is given by Definition 3. For simplicity of presentation, we postulate, in addition, that the event $\left\{\tau_{C}=\tau_{I}\right\}$ is negligible under $\mathbb{Q}^{r}$ and thus the close-out payoff $\vartheta_{\tau}$ is given by (6) with $Q$ given by (31). Then, we have following result, which is a consequence of Corollary 2 and Definition 12.

We stress once again that our goal in this subsection is not merely to compute the price of a contract or to simply compare the actual price of a contract with its clean price but, more importantly, to explicitly identify the individual contribution of each cost and risk to the overall price of a contract. This explains why in Proposition 2 we use the probability measure $\mathbb{Q}^{r}$, as opposed to the probability measure $\mathbb{Q}^{f, h, f}$ employed in Corollary 1. The advantage of (34) over (27) is that the former representation gives more information about the intricacies of the multi-curve pricing mechanism and, at the same time, it uses a fixed probability measure $\mathbb{Q}^{r}$, which does not change with the current market conditions. However, we recall that (34) is in fact a linear BSDE, not just a simple conditional expectation, and thus its solution requires more steps.

Proposition 2. Under the risk-free close-out valuation $Q$ given by (31), the ex-dividend price for the collateralized defaultable contract $(A, R, C, \tau)$ equals, on the event $\{t<\tau\}$ for every $t \in[0, T]$,

$$
\begin{aligned}
& \pi_{t}(A, R, C, \tau)=\pi_{t}^{r}(A)+\mathbb{E}_{\mathbb{Q}^{r}}\left(D(t, \tau) \mathbb{1}_{\{\tau \leq T\}}\left(\mathbb{1}_{\left\{\tau_{C}<\tau_{I}\right\}} L_{C} Y^{+}-\mathbb{1}_{\left\{\tau_{I}<\tau_{C}\right\}} L_{I} Y^{-}\right) \mid \mathcal{G}_{t}\right) \\
& +\mathbb{E}_{\mathbb{Q}^{r}}\left(\int_{t}^{\widehat{\tau}} D(t, u)\left(r_{u}-f_{u}\right) F_{u} d u+\sum_{i=1}^{d} \int_{t}^{\widehat{\tau}} D(t, u)\left(r_{u}-h_{u}^{i}\right) F_{u}^{i} d u \mid \mathcal{G}_{t}\right) \\
& +\mathbb{E}_{\mathbb{Q}^{r}}\left(\int_{t}^{\widehat{\tau}} D(t, u)\left(\bar{c}_{u}-r_{u}\right) C_{u} d u \mid \mathcal{G}_{t}\right) .
\end{aligned}
$$

Proof. In view of Definition 12, to obtain (34) from (28) and (6), it suffices to observe that, on the event $\{t<\tau\}$,

$$
\int_{] t, \widehat{\tau}]}\left(B_{u}^{r}\right)^{-1} d \widetilde{A}_{u}=\int_{] t, T]}\left(B_{u}^{r}\right)^{-1} d A_{u}-\mathbb{1}_{\{\tau \leq T\}} \int_{[\tau, T]}\left(B_{u}^{r}\right)^{-1} d A_{u}
$$

and thus

$$
\begin{aligned}
\mathbb{E}_{\mathbb{Q}^{r}}\left(-\int_{t t, \hat{\tau}]} D(t, u) d \widetilde{A}_{u} \mid \mathcal{G}_{t}\right) & =\mathbb{E}_{\mathbb{Q}^{r}}\left(-\int_{] t, T]} D(t, u) d A_{u}+\mathbb{1}_{\{\tau \leq T\}} \int_{[\tau, T]} D(t, u) d A_{u} \mid \mathcal{G}_{t}\right) \\
& =\pi_{t}^{r}(A)+\mathbb{E}_{\mathbb{Q}^{r}}\left(\mathbb{1}_{\{\tau \leq T\}} Q_{\tau} \mid \mathcal{G}_{t}\right)
\end{aligned}
$$

where the last equality follows from (31) and (32).

Representation (34) of the investor's ex-dividend price for $(A, R, C, \tau)$ can be given the following financial interpretation:

$$
\pi_{t}(A, R, C, \tau)=\pi_{t}^{r}(A)+\mathrm{CVA}_{t}-\mathrm{DVA}_{t}+\mathrm{FVA}_{t}^{f}+\sum_{i=1}^{d} \mathrm{FVA}_{t}^{h^{i}}+\mathrm{LVA}_{t}
$$

where the credit valuation adjustment $\mathrm{CVA}_{t}$ equals

$$
\mathrm{CVA}_{t}=\mathbb{E}_{\mathbb{Q}^{r}}\left(D(t, \tau) \mathbb{1}_{\{\tau \leq T\}} \mathbb{1}_{\left\{\tau_{C}<\tau_{I}\right\}} L_{C} \mathrm{Y}^{+} \mid \mathcal{G}_{t}\right),
$$

the debit valuation adjustment $\mathrm{DVA}_{t}$ equals

$$
\mathrm{DVA}_{t}=\mathbb{E}_{\mathbb{Q}^{r}}\left(D(t, \tau) \mathbb{1}_{\{\tau \leq T\}} \mathbb{1}_{\left\{\tau_{I}<\tau_{C}\right\}} L_{I} \mathrm{Y}^{-} \mid \mathcal{G}_{t}\right),
$$


the treasury funding valuation adjustment $\mathrm{FVA}_{t}^{f}$ is given by

$$
\mathrm{FVA}_{t}^{f}=\mathbb{E}_{\mathbb{Q}^{r}}\left(\int_{t}^{\widehat{\tau}} D(t, u)\left(r_{u}-f_{u}\right) F_{u} d u \mid \mathcal{G}_{t}\right),
$$

the repo funding valuation adjustments $\mathrm{FVA}_{t}^{h^{i}}$ for $i=1,2, \ldots, d$ are given by

$$
\mathrm{FVA}_{t}^{h^{i}}=\mathbb{E}_{\mathbb{Q}^{r}}\left(\int_{t}^{\widehat{\tau}} D(t, u)\left(r_{u}-h_{u}^{i}\right) F_{u}^{i} d u \mid \mathcal{G}_{t}\right)
$$

and liquidity valuation adjustment $\mathrm{LVA}_{t}$ satisfies

$$
\operatorname{LVA}_{t}=\mathbb{E}_{\mathbb{Q}^{r}}\left(\int_{t}^{\hat{\tau}} D(t, u)\left(\bar{c}_{u}-r_{u}\right) C_{u} d u \mid \mathcal{G}_{t}\right) .
$$

Notice that the valuation adjustments given above can be computed independently, provided that the processes $F$ and $F^{i}$ are known. The latter processes can be found by solving the linear BSDE, which is a special case of the pricing BSDE given in Proposition 4.

\section{Nonlinear Markets with Funding Costs and Default Risk}

A nonlinear extension of the linear framework introduced in the preceding section is obtained when a single cash rate $f$ is replaced by differential lending and borrowing rates, denoted as $f^{l}$ and $f^{b}$, respectively, and, similarly, by introducing differential repo rates for long and short positions in the stock $S^{i}$, which are denoted as $h^{i, l}$ and $h^{i, b}$, respectively. Then, we obtain the following representation of the value process $V^{p}\left(\varphi, A^{R, C}\right)$ of a portfolio generated by strategy $\varphi$

$$
V_{t}^{p}\left(\varphi, A^{R, C}\right)=\psi_{t}^{l} B_{t}^{l}+\psi_{t}^{b} B_{t}^{i, b}+\sum_{j=1}^{2} \kappa_{t}^{j} Z^{j}(t, T)+\sum_{i=1}^{d}\left(\psi_{t}^{i, l} B_{t}^{i, l}+\psi_{t}^{i, b} B_{t}^{i, b}+\xi_{t}^{i} S_{t}^{i}\right)+\sum_{i=d+1}^{m} \xi_{t}^{i} S_{t}^{i}
$$

where, consistently with the financial interpretation of trading within the nonlinear framework, we postulate that $\psi_{t}^{l} \geq 0, \psi_{t}^{b} \leq 0$ and $\psi_{t}^{l} \psi_{t}^{b}=0$ for all $t \in[0, T]$. Similarly, we assume that $\psi_{t}^{i, l} \geq 0, \psi_{t}^{i, b} \leq 0$ and $\psi_{t}^{i, l} \psi_{t}^{i, b}=0$ for $i=1,2, \ldots, d$ and, in addition, we impose the repo trading condition for risky assets $S^{1}, S^{2}, \ldots, S^{d}$

$$
\psi_{t}^{i, l} B_{t}^{i, l}+\psi_{t}^{i, b} B_{t}^{i, b}+\xi_{t}^{i} S_{t}^{i}=0 .
$$

For convenience, we denote

$$
\begin{aligned}
F_{t} & =\psi_{t}^{l} B_{t}^{l}+\psi_{t}^{b} B_{t}^{b}=V_{t}^{p}\left(\varphi, A^{R, C}\right)-\sum_{j=1}^{2} \kappa_{t}^{j} Z^{j}(t, T)-\sum_{i=d+1}^{m} \xi_{t}^{i} S_{t}^{i} \\
& =V_{t}^{p}\left(\varphi, A^{R, C}\right)-\sum_{j=1}^{2} Z_{t}^{j}-\sum_{i=d+1}^{m} H_{t}^{i}
\end{aligned}
$$

where $Z_{t}^{j}=\kappa_{t}^{j} Z^{j}(t, T)$ for $j=1,2$ and $H_{t}^{i}=\xi_{t}^{i} S_{t}^{i}=-F_{t}^{i}$ for $i=d+1, d+2, \ldots, m$.

\subsection{Nonlinear Dynamics of the Value Process of a Trading Strategy}

We are now in a position to derive the nonlinear dynamics of the value process for a collateralized defaultable contract $(A, R, C, \tau)$. Recall that $\tau=\tau_{I} \wedge \tau_{C}$ so that $\widehat{\tau}:=\tau \wedge T$ is the effective maturity of the contract.

Lemma 3. We have $\psi_{t}^{l}=\left(B_{t}^{l}\right)^{-1} F_{t}^{+}, \psi_{t}^{b}=-\left(B_{t}^{b}\right)^{-1} F_{t}^{-}$and for $i=1,2, \ldots, d$

$$
\psi_{t}^{i, l}=\left(B_{t}^{i, l}\right)^{-1}\left(H_{t}^{i}\right)^{-}=\left(B_{t}^{i, l}\right)^{-1}\left(F_{t}^{i}\right)^{+}, \quad \psi_{t}^{i, b}=-\left(B_{t}^{i, b}\right)^{-1}\left(H_{t}^{i}\right)^{+}=-\left(B_{t}^{i, b}\right)^{-1}\left(F_{t}^{i}\right)^{-} .
$$


Proof. It suffices to note that

$$
\psi_{t}^{l} B_{t}^{l}+\psi_{t}^{b} B_{t}^{b}=F_{t}, \quad \psi_{t}^{i, l} B_{t}^{i, l}+\psi_{t}^{i, b} B_{t}^{i, b}=-\xi_{t}^{i} S_{t}^{i}=-H_{t}^{i}=F_{t}^{i}
$$

and to make use of the postulated conditions.

Lemma 4. The value process $V^{p}\left(\varphi, A^{R, C}\right)$ of a self-financing trading strategy associated with collateralized defaultable contract $(A, R, C, \tau)$ satisfies on $[0, \widehat{\tau}]$

$$
\begin{aligned}
d V_{t}^{p}\left(\varphi, A^{R, C}\right)= & f_{t}^{l} F_{t}^{+} d t-f_{t}^{b} F_{t}^{-} d t+\sum_{j=1}^{2} \kappa_{t}^{j} d Z^{j}(t, T) \\
& +\sum_{i=1}^{d}\left(\xi_{t}^{i} d S_{t}^{i}+h_{t}^{i, l}\left(F_{t}^{i}\right)^{+} d t-h_{t}^{i, b}\left(F_{t}^{i}\right)^{-} d t\right) \\
& +\sum_{i=d+1}^{m} \xi_{t}^{i} d S_{t}^{i}+d \widetilde{A}_{t}+d \widetilde{C}_{t}-\bar{c}_{t} C_{t} d t+d\left(\mathbb{1}_{\{t \geq \tau\}} R_{\tau}\right)
\end{aligned}
$$

where $\bar{c}$ is given by (12), $\widetilde{A}$ and $\widetilde{C}$ are given by (23) and $R_{\tau}$ is given by (3).

Proof. By a minor extension of Definition 7 , the self-financing condition for $\varphi$ reads, for $t \in[0, \widehat{\tau}]$

$$
\begin{aligned}
d V_{t}^{p}\left(\varphi, A^{R, C}\right)= & \psi_{t}^{l} d B_{t}^{l}+\psi_{t}^{b} d B_{t}^{b}+\sum_{j=1}^{2} \kappa_{t}^{j} d Z^{j}(t, T)+\sum_{i=1}^{d}\left(\psi_{t}^{i, l} d B_{t}^{i, l}+\psi_{t}^{i, b} d B_{t}^{i, b}+\xi_{t}^{i} d S_{t}^{i}\right) \\
& +\sum_{i=d+1}^{m} \xi_{t}^{i} d S_{t}^{i}+d A_{t}^{R, C}
\end{aligned}
$$

where $A^{R, C}$ is given by (15). Therefore, in view of Lemma 3, we obtain the following expression for the dynamics of the value process:

$$
\begin{aligned}
d V_{t}^{p}\left(\varphi, A^{R, C}\right)= & \left(B_{t}^{l}\right)^{-1} F_{t}^{+} d B_{t}^{l}-\left(B_{t}^{b}\right)^{-1} F_{t}^{-} d B_{t}^{b}+\sum_{j=1}^{2} \kappa_{t}^{j} d Z^{j}(t, T)+\sum_{i=d+1}^{m} \xi_{t}^{i} d S_{t}^{i} \\
& +\sum_{i=1}^{d}\left(B_{t}^{i, l}\right)^{-1}\left(F_{t}^{i}\right)^{+} d B_{t}^{i, l}-\sum_{i=1}^{d}\left(B_{t}^{i, b}\right)^{-1}\left(F_{t}^{i}\right)^{-} d B_{t}^{i, b}+\sum_{i=1}^{d} \xi_{t}^{i} d S_{t}^{i}+d A_{t}^{R, C} .
\end{aligned}
$$

Under the assumption of absolute continuity of cash/repo accounts, using also (15) and (23), we obtain (36).

To obtain a convenient representation of the dynamics of $V^{p}\left(\varphi, \widetilde{A}^{C}\right)$, we introduce the effective funding rate, $\bar{f}$, and the effective repo rates, $\bar{h}^{i}$. It is obvious that, in general, the effective rates $\bar{f}$ and $\bar{h}^{i}$ depend in a nonlinear way on investor's strategy $\varphi$ through processes $F$ and $F^{i}$.

Lemma 5. The process $V^{p}\left(\varphi, \widetilde{A}^{C}\right)$ satisfies on $[0, \widehat{\tau}]$

$$
d V_{t}^{p}\left(\varphi, \widetilde{A}^{C}\right)=\left(\bar{f}_{t} F_{t}+\bar{h}_{t}^{i} F_{t}^{i}-\bar{c}_{t} C_{t}\right) d t+\sum_{j=1}^{2} \kappa_{t}^{j} d Z^{j}(t, T)+\sum_{i=1}^{m} z_{t}^{i} d S_{t}^{i}+d \widetilde{A}_{t}+d \widetilde{C}_{t}
$$

where the effective funding rate $\bar{f}$ equals

$$
\bar{f}_{t}:=f_{t}^{l} \mathbb{1}_{\left\{F_{t} \geq 0\right\}}+f_{t}^{b} \mathbb{1}_{\left\{F_{t}<0\right\}}
$$

and the effective repo rates $\bar{h}^{i}, i=1,2, \ldots, d$ are given by

$$
\bar{h}_{t}^{i}:=h_{t}^{i, l} \mathbb{1}_{\left\{F_{t}^{i} \geq 0\right\}}+h_{t}^{i, b} \mathbb{1}_{\left\{F_{t}^{i}<0\right\}} .
$$




\subsection{Nonlinear Probabilistic Valuation Formula}

Recall that

$$
B_{t}^{\eta}:=\exp \left(\int_{0}^{t} \eta_{u} d u\right), \quad B_{t}^{\zeta^{j}}:=\exp \left(\int_{0}^{t} \zeta_{u}^{j} d u\right), \quad B_{t}^{\gamma^{i}}:=\exp \left(\int_{0}^{t} \gamma_{u}^{i} d u\right)
$$

where $\eta, \zeta^{1}, \zeta^{2}, \gamma^{i}, i=1,2, \ldots, d$ and $v^{i}, i=d+1, d+2, \ldots, m$ are arbitrary adapted and integrable processes. Let $\mathbb{Q}^{\zeta, \gamma, v}$ be a probability measure such that the processes $\left(B^{\zeta^{j}}\right)^{-1} Z^{j}(t, T), j=1,2,\left(B^{\gamma^{i}}\right)^{-1} S^{i}, i=1,2, \ldots, d$ and $\left(B^{v^{i}}\right)^{-1} S^{i}, i=d+1, d+2, \ldots, m$ are $\mathbb{Q}^{\zeta, \gamma, v}$-local martingales.

By combining Lemma 2 with Lemma 5 we obtain the following result, which is a nonlinear counterpart of Theorem 1 . To ensure the validity of this result, one needs to impose some mild integrability assumptions. Recall from Definition 3 that the CSA closeout payoff satisfies $\vartheta_{\tau}=R_{\tau}+C_{\tau-}$.

Theorem 2. Assume that the collateralized defaultable contract $(A, R, C, \tau)$ can be replicated by a trading strategy $\varphi$ and the associated process $\bar{V}^{\eta}\left(\varphi, \widetilde{A}^{C}\right)$ is a true martingale under $\mathbb{Q}^{\zeta, \gamma, v}$. Then, $V_{t}^{p}\left(\varphi, A^{R, C}\right)$ equals, on the event $\{t<\tau\}$ for every $t \in[0, T]$,

$$
\begin{aligned}
V_{t}^{p}\left(\varphi, A^{R, C}\right) & =\mathbb{E}_{\mathbb{Q} \zeta, \gamma, v}\left(-\int_{] t, \widehat{\tau}]} D^{\eta}(t, u) d \widetilde{A}_{u}^{C}+D^{\eta}(t, T) C_{T} \mathbb{1}_{\{\tau>T\}}-D^{\eta}(t, \tau) R_{\tau} \mathbb{1}_{\{\tau \leq T\}} \mid \mathcal{G}_{t}\right) \\
& +\mathbb{E}_{\mathbb{Q}^{\zeta, \gamma, v}}\left(\int_{t}^{\widehat{\tau}} D^{\eta}(t, u)\left(\eta_{u}-\bar{f}_{u}\right) F_{u} d u+\sum_{j=1}^{2} \int_{t}^{\widehat{\tau}} D^{\eta}(t, u)\left(\eta_{u}-\zeta_{u}^{j}\right) Z_{u}^{j} d u \mid \mathcal{G}_{t}\right) \\
& +\mathbb{E}_{\mathbb{Q}^{\zeta, \gamma, v}}\left(\sum_{i=1}^{d} \int_{t}^{\widehat{\tau}} D^{\eta}(t, u)\left(\bar{h}_{u}^{i}-\gamma_{u}^{i}\right) H_{u}^{i} d u+\sum_{i=d+1}^{m} \int_{t}^{\widehat{\tau}} D^{\eta}(t, u)\left(\eta_{u}-v_{u}^{i}\right) H_{u}^{i} d u \mid \mathcal{G}_{t}\right) .
\end{aligned}
$$

The ex-dividend price for the collateralized defaultable contract $(A, R, C, \tau)$ is given by, on the event $\{t<\tau\}$, for every $t \in[0, T]$,

$$
\begin{aligned}
\pi_{t}(A, R, C, \tau) & =\mathbb{E}_{\mathbb{Q}^{\zeta, \gamma, v}}\left(-\int_{] t, \widehat{\tau}]} D^{\eta}(t, u) d \widetilde{A}_{u}-D^{\eta}(t, \tau) \vartheta_{\tau} \mathbb{1}_{\{\tau \leq T\}}+\int_{t}^{\widehat{\tau}} D^{\eta}(t, u)\left(\eta_{u}-\bar{f}_{u}\right) F_{u} d u \mid \mathcal{G}_{t}\right) \\
& +\mathbb{E}_{\mathbb{Q} \zeta, \gamma, v}\left(\sum_{j=1}^{2} \int_{t}^{\widehat{\tau}} D^{\eta}(t, u)\left(\eta_{u}-\zeta_{u}^{j}\right) Z_{u}^{j} d u+\sum_{i=1}^{d} \int_{t}^{\widehat{\tau}} D^{\eta}(t, u)\left(\bar{h}_{u}^{i}-\gamma_{u}^{i}\right) H_{u}^{i} d u \mid \mathcal{G}_{t}\right) \\
& +\mathbb{E}_{\mathbb{Q} \zeta, \gamma, v}\left(\sum_{i=d+1}^{m} \int_{t}^{\widehat{\tau}} D^{\eta}(t, u)\left(\eta_{u}-v_{u}^{i}\right) H_{u}^{i} d u+\int_{t}^{\widehat{\tau}} D^{\eta}(t, u)\left(\bar{c}_{u}-\eta_{u}\right) C_{u} d u \mid \mathcal{G}_{t}\right)
\end{aligned}
$$

where the effective rates $\bar{f}, \bar{h}^{i}$ and $\bar{c}$ are given by (38), (39), and (12), respectively.

Proof. In view of Lemma 5, the proof of Theorem 2 is analogous to the proof of Theorem 1 and thus it is omitted.

\subsection{Nonlinear Valuation with XVAs under Risk-Free Close-Out}

Theorem 2 can be used to identify contributions of various adjustments to the investor's price with respect to the clean price computed using the non-accessible short-term rate. To this end, one can use the following consequence of Theorem 2, which corresponds to Corollary 2. Recall that we use the shorthand notation $\mathbb{Q}^{r}=\mathbb{Q}^{r, r, r}$. As in Section 3.2, we assume that the event $\left\{\tau_{C}=\tau_{I}\right\}$ is negligible under $\mathbb{Q}^{r}$ and thus, without loss of generality, the CSA close-out payoff $\vartheta_{\tau}$ is assumed to be given by (6).

Corollary 3. The ex-dividend price for the collateralized defaultable contract $(A, R, C, \tau)$ satisfies, on the event $\{t<\tau\}$ for every $t \in[0, T]$, 


$$
\begin{aligned}
\pi_{t}(A, R, C, \tau) & =\mathbb{E}_{\mathbb{Q}^{r}}\left(-\int_{] t, \widehat{\tau}]} D(t, u) d \widetilde{A}_{u}-D(t, \tau) \vartheta_{\tau}(R, C) \mathbb{1}_{\{\tau \leq T\}}+\int_{t}^{\widehat{\tau}} D(t, u)\left(r_{u}-\bar{f}_{u}\right) F_{u} d u \mid \mathcal{G}_{t}\right) \\
& +\mathbb{E}_{\mathbb{Q}^{r}}\left(\sum_{i=1}^{d} \int_{t}^{\widehat{\tau}} D(t, u)\left(r_{u}-\bar{h}_{u}^{i}\right) F_{u}^{i} d u+\int_{t}^{\widehat{\tau}} D(t, u)\left(\bar{c}_{u}-r_{u}\right) C_{u} d u \mid \mathcal{G}_{t}\right) .
\end{aligned}
$$

This recovers and generalizes to the market with several accounts the "adjusted cash flow" valuation formula (5).

We maintain the assumption made in Section 3.2 about the exact specification of the CSA close-out payoff $\vartheta$. Recall also that the case of the joint defaults was precluded. Then, Proposition 2 can be easily extended to cover the case of the nonlinear setup. It suffices to replace $f, h^{i}$ and $c$ by $\bar{f}, \bar{h}^{i}$ and $\bar{c}$, respectively, in Equation (34). The proof of the next result is analogous to the proof of Proposition 2 and thus it is omitted.

Proposition 3. Under the risk-free close-out valuation $Q$ given by (31), the ex-dividend price for the collateralized defaultable contract $(A, R, C, \tau)$ equals, on the event $\{t<\tau\}$, for every $t \in[0, T]$,

$$
\begin{aligned}
& \pi_{t}(A, R, C, \tau)=\pi_{t}^{r}(A)+\mathbb{E}_{\mathbb{Q}^{r}}\left(D(t, \tau) \mathbb{1}_{\{\tau \leq T\}}\left(\mathbb{1}_{\left\{\tau_{C}<\tau_{I}\right\}} L_{C} Y^{+}-\mathbb{1}_{\left\{\tau_{I}<\tau_{C}\right\}} L_{I} Y^{-}\right) \mid \mathcal{G}_{t}\right) \\
& +\mathbb{E}_{\mathbb{Q}^{r}}\left(\int_{t}^{\widehat{\tau}} D(t, u)\left(r_{u}-\bar{f}_{u}\right) F_{u} d u+\sum_{i=1}^{d} \int_{t}^{\widehat{\tau}} D(t, u)\left(r_{u}-\bar{h}_{u}^{i}\right) F_{u}^{i} d u \mid \mathcal{G}_{t}\right) \\
& +\mathbb{E}_{\mathbb{Q}^{r}}\left(\int_{t}^{\widehat{\tau}} D(t, u)\left(\bar{c}_{u}-r_{u}\right) C_{u} d u \mid \mathcal{G}_{t}\right) .
\end{aligned}
$$

Proposition 3 leads in turn to the following formal decomposition of the ex-dividend price for $(A, R, C, \tau)$ into its clean price and valuation adjustments (for their interpretation, see Section 3.2), which holds on the event $\{t<\tau\}$ for every $t \in[0, T]$,

$$
\pi_{t}(A, R, C, \tau)=\pi_{t}^{r}(A)+\mathrm{CVA}_{t}-\mathrm{DVA}_{t}+\mathrm{FVA}_{t}^{\bar{f}}+\sum_{i=1}^{d} \mathrm{FVA}_{t}^{\bar{h}^{i}}+\mathrm{LVA}_{t} .
$$

We stress that an apparent additivity property of valuation adjustments in (44) is illusory since the terms in the right-hand side of (44) cannot be computed independently since, in fact, to compute the price given by (43), and hence, to obtain the price decomposition (44), one needs to solve the nonlinear BSDE for the price and replicating strategy, which is formally stated in Proposition 4.

\subsection{Nonlinear Pricing BSDE}

As opposed to the linear market framework of Section 3.1, Theorem 2, Corollary 3, and Proposition 3 do not furnish closed-form expressions for the price, since their righthand sides depend in a nonlinear way on several unknown processes. In fact, the pricing equations from those results should be interpreted as nonlinear BSDEs and thus their solutions are not known explicitly and require suitable numerical method. Under the standing assumption that the investor's initial endowment is null, Equation (37) yields the nonlinear BSDE (45) for the portfolio's value $Y:=V^{p}\left(\varphi, \widetilde{A}^{C}\right)$ and hedge ratios $(\kappa, \xi)$, whereas all other components of the replicating strategy $\varphi$ can be found using Lemma 3 . Then, the equality $\pi_{t}(A, R, C, \tau)=Y_{t}-C_{t}$ holds on the event $\{t<\tau\}$ for every $t \in[0, T]$.

Proposition 4. The portfolio's value $Y=V^{p}\left(\varphi, \widetilde{A}^{C}\right)$ and the hedge ratios $(\kappa, \xi)$ satisfy the backward stochastic differential equation, as follows:

$$
d Y_{t}=\left(\bar{f}_{t} \widehat{Y}_{t}-\sum_{i=1}^{d} \bar{h}_{t}^{i} \xi_{t}^{i} S_{t}^{i}-\bar{c}_{t} C_{t}\right) d t+\sum_{j=1}^{2} \kappa_{t}^{j} d Z^{j}(t, T)+\sum_{i=1}^{m} \xi_{t}^{i} d S_{t}^{i}+d \widetilde{A}_{t}+d \widetilde{C}_{t}
$$


where

$$
\widehat{Y}_{t}:=Y_{t}-\sum_{j=1}^{2} \kappa_{t}^{j} Z^{j}(t, T)-\sum_{i=d+1}^{m} \xi_{t}^{i} S_{t}^{i}
$$

and from (38) and (39)

$$
\begin{aligned}
& \bar{f}_{t}:=f_{t}^{l} \mathbb{1}_{\left\{\widehat{Y}_{t} \geq 0\right\}}+f_{t}^{b} \mathbb{1}_{\left\{\widehat{Y}_{t}<0\right\}}, \\
& \bar{h}_{t}^{i}:=h_{t}^{i, l} \mathbb{1}_{\left\{\xi_{t}^{i} S_{t}^{i}<0\right\}}+h_{t}^{i, b} \mathbb{1}_{\left\{\xi_{t}^{i} S_{t}^{i} \geq 0\right\}^{\prime}} \\
& \bar{c}_{t}:=c_{t}^{l} \mathbb{1}_{\left\{C_{t}<0\right\}}+c_{t}^{b} \mathbb{1}_{\left\{C_{t} \geq 0\right\}} .
\end{aligned}
$$

The terminal condition at $\widehat{\tau}$ reads $Y_{\widehat{\tau}}=\mathbb{1}_{\{\tau>T\}} C_{T}-\mathbb{1}_{\{\tau \leq T\}} R_{\tau}$ where the recovery payoff $R_{\tau}$ equals

$$
R_{\tau}=\mathbb{1}_{\left\{\tau_{C}<\tau_{I}\right\}}\left(R_{C} \mathrm{Y}^{+}-\mathrm{Y}^{-}\right)+\mathbb{1}_{\left\{\tau_{I}<\tau_{C}\right\}}\left(\mathrm{Y}^{+}-R_{I} \mathrm{Y}^{-}\right)
$$

where $\mathrm{Y}=Q_{\tau}-C_{\tau-}$.

Given any specific semimartingale model for risky assets and any funding costs, it is usually possible to show that the pricing BSDE has a unique solution in a suitable space of stochastic processes. For instance, Nie and Rutkowski [7-9] studied pricing and hedging BSDEs for a contract $(A, C)$ (so defaults were not considered) with both the exogenous and endogenous collateralization whereas Bichuch et al. [1] included also the default risk. For pricing BSDEs with a single jump martingale, the reader is referred to Crépey [5,6], Crépey and Song [47], Dumitrescu et al. [48], and Peng and Xu [49], and for BSDEs driven by discontinuous martingales, we refer the reader to Carbone et al. [50] and Nie and Rutkowski [51].

\section{Incomplete Market with Funding Benefit at Default}

In the "adjusted cash flows" approach, the derivation was agnostic of any replication, while in the "replication approach", the concept of replication is clearly an integral part. With this in mind, we ask ourselves whether we can reconcile these two in a more general setting of an incomplete market where we relax the replication assumption and we postulate instead that the properly deflated investor's gains process is a martingale. We show in this section what pricing means and present conditions under which the net funding benefit of the issuer of the contract vanishes, rendering the knowledge of the specific hedging strategy unnecessary for computation of valuation adjustments.

In the previous section, we have assumed that the contract that we are evaluating can be replicated. Now we wish to examine briefly what happens if this assumption is relaxed. We will concentrate here on the case where the contract is subject to defaults of both the bank (investor) and the counterparty, while the bank cannot trade its own or counterparty's bonds (i.e., we postulate that $\kappa^{1}=\kappa^{2}=0$ ). Of course, this means that we deal with an incomplete market model in which some contracts cannot be replicated and thus other means should by employed to assess the marked-to-market value of a contract.

To further simplify the setup, we suppose that all of the hedging instruments, defaultfree bonds and equities are traded on the repo market (i.e., we set $d=m$ ), and that repo rates are the same across all positions and directions of the trade (i.e., we assume that $h^{i, l}=h^{i, b}=h$ for all $i$ ). Furthermore, we postulate that the borrowing account $B^{b}$ satisfies

$$
d B_{t}^{b}=f_{t}^{b} B_{t}^{b} d t-L_{I} B_{t-}^{b} d \mathbb{1}_{\left\{t \geq \tau_{I}\right\}}
$$

which models the property that at the bank's default only a fraction $R_{I}=1-L_{I}$ of the bank's debt will be repaid to its creditors. The same recovery rate $R_{I}$ applies to the amount due to the counterparty at time of investors default. Since we are including default in our model, we will also make use of Equations (14) and (15) to define the stream of cash flows generated by the contract. In order to mark to market a contract that cannot be replicated, we find it convenient to extend slightly the definition of trading strategy of 
Section 3 by including the contract itself. To be more specific, we are interested in buyand-hold strategies $\varphi$ of the form $\left(1, \psi_{t}^{l}, \psi_{t}^{b}, \psi^{1}, \ldots, \psi^{d}, \xi^{1}, \ldots, \xi^{d}\right)$, where 1 means that one unit of the derivative asset with the promised cash flows $A$ is held by the investor till its effective maturity. Notice that, if the investor has sold at time 0 a call or put option, then the marked-to-market value of their position is negative for $t>0$ (it is simply equal to the negative of the price at time $t$ in the classical linear arbitrage-free framework).

We denote by $W\left(\varphi, A^{R, C}\right)$ and $W^{p}\left(\varphi, A^{R, C}\right)$ the investor's wealth and the marked-tomarket value of their portfolio of assets, respectively, so that on the event $\{t<\tau\}$,

$$
W_{t}^{p}\left(\varphi, A^{R, C}\right)=\widehat{\pi}_{t}+\psi_{t}^{l} B_{t}^{l}+\psi_{t}^{b} B_{t}^{b}+\sum_{i=1}^{d}\left(\psi_{t}^{i} B_{t}^{i}+\xi_{t}^{i} S_{t}^{i}\right)=\widehat{\pi}_{t}+\psi_{t}^{l} B_{t}^{l}+\psi_{t}^{b} B_{t}^{b}=\widehat{\pi}_{t}+F_{t}
$$

and $W_{t}\left(\varphi, A^{R, C}\right)=W_{t}^{p}\left(\varphi, A^{R, C}\right)-C_{t}$, where $\widehat{\pi}_{t}$ is the shorthand for the market-to-market value, $\widehat{\pi}_{t}(A, R, C, \tau)$, and where we used the equality $\psi_{t}^{i} B_{t}^{i}+\xi_{t}^{i} S_{t}^{i}=0$. Recall that the convention regarding the collateral is that $C_{t}>0$ means that the investor receives the collateral, while $C_{t}<0$ means that the investor posts it. Hence the minus sign in front of the collateral account is needed since the wealth $W_{t}\left(\varphi, A^{R, C}\right)$ is what the investor would obtain at time $t$ if the investor were to liquidate every asset owned. At default time, the portfolio's value and the investor's wealth satisfy, on the event $\{\tau \leq T\}$,

$$
W_{\tau}^{p}\left(\varphi, A^{R, C}\right)=W_{\tau}\left(\varphi, A^{R, C}\right)=R_{\tau}+\psi_{\tau}^{l} B_{\tau}^{l}+\psi_{\tau}^{b} B_{\tau}^{b} .
$$

In essence, our valuation arguments in this subsection are based on the premise that the extended market model where the contract already held by the investor should remain arbitrage-free, as seen from their perspective. More specifically, we will assume that the contract is already held by the investor and we search for the process $\hat{\pi}$, such that the suitably deflated investor's wealth has the martingale property under a chosen martingale measure. Notice that the convention regarding the sign of $\hat{\pi}$ is different from the one previously adopted in Sections 2 and 3 where we examined the investor's unilateral selling price for the contract at time $t$, since the process $\widehat{\pi}$ now represents the marked-to-market value of the contract held by the investor.

Definition 13. The net gains process of the investor's portfolio is given by, on $[0, \widehat{\tau}]$,

$$
\begin{aligned}
G_{t} & =\mathbb{1}_{\{t<\tau\}} \widehat{\pi}_{t}+\int_{0}^{t \wedge \tau} \psi_{u}^{l} d B_{u}^{l}+\int_{0}^{t \wedge \tau} \psi_{u-}^{b} d B_{u}^{b}+\sum_{i=1}^{d}\left(\int_{0}^{t \wedge \tau} \xi_{u}^{i} d S_{u}^{i}+\int_{0}^{t \wedge \tau} \psi_{u}^{i} d B_{u}^{i}\right) \\
& +\int_{0}^{t} d A_{u}^{R, C}-\mathbb{1}_{\{t<\tau\}} C_{t}
\end{aligned}
$$

where

$$
d A_{u}^{R, C}=d \widetilde{A}_{u}+d \widetilde{C}_{u}-\mathbb{1}_{\{u<\tau\}} \bar{c}_{u} C_{u} d u+R_{\tau} d\left(\mathbb{1}_{\{u \geq \tau\}}\right)
$$

Recall that $\widetilde{A}_{t}=\mathbb{1}_{\{t<\tau\}} A_{t}+\mathbb{1}_{\{t \geq \tau\}} A_{\tau-}$ and $\widetilde{C}_{t}=\mathbb{1}_{\{t<\tau\}} C_{t}+\mathbb{1}_{\{t \geq \tau\}} C_{\tau-}$. We consider only self-financing strategies, that is, those satisfying on $[0, \widehat{\tau}]$

$$
W_{t}\left(\varphi, A^{R, C}\right)=W_{0}\left(\varphi, A^{R, C}\right)+G_{t}-G_{0} .
$$

Let us denote by $\eta$ the prevailing deposit rate at which the bank may lend cash to other entities. For the investor's deflated wealth, $W_{t}^{\eta}\left(\varphi, A^{R, C}\right):=\left(B_{t}^{\eta}\right)^{-1} W_{t}\left(\varphi, A^{R, C}\right)$, we obtain

$$
W_{t}^{\eta}\left(\varphi, A^{R, C}\right)=W_{0}^{\eta}\left(\varphi, A^{R, C}\right)+G_{t}^{\eta}-G_{0}^{\eta}
$$

where the deflated net gains process $G_{u}^{\eta}$ satisfies 


$$
\begin{aligned}
d G_{u}^{\eta} & =\left(B_{u}^{\eta}\right)^{-1} d \widehat{\pi}_{u}+\left(B_{u}^{\eta}\right)^{-1} \psi_{u}^{l} d B_{u}^{l}+\left(B_{u}^{\eta}\right)^{-1} \psi_{u-}^{b} d B_{u}^{b}+\sum_{i=1}^{d}\left(B_{u}^{\eta}\right)^{-1}\left(\xi_{u}^{i} d S_{u}^{i}+\psi_{u}^{i} d B_{u}^{i}\right) \\
& -\left(B_{u}^{\eta}\right)^{-1} \mathbb{1}_{\{u<\tau\}} \bar{c}_{u} C_{u} d u+\left(B_{u}^{\eta}\right)^{-1} d \widetilde{A}_{u}+\left(B_{u}^{\eta}\right)^{-1} C_{\tau-} d\left(\mathbb{1}_{\{t \geq \tau\}}\right)+\left(B_{u}^{\eta}\right)^{-1} d\left(\mathbb{1}_{\{t \geq \tau\}} R_{\tau}\right) \\
& -\eta_{u}\left(B_{u}^{\eta}\right)^{-1}\left(\widehat{\pi}_{u}+\psi_{u}^{l} B_{u}^{l}+\psi_{u}^{b} B_{u}^{b}\right) d u+\eta_{u}\left(B_{u}^{\eta}\right)^{-1} \mathbb{1}_{\{u<\tau\}} C_{u} d u
\end{aligned}
$$

To proceed further, we henceforth postulate that the process $G^{\eta}$ is a martingale stopped at $\widehat{\tau}$ with respect to the filtration $\mathbb{G}$ and under a probability measure $\mathbb{Q}^{h}$, which can be identified with the probability measure $\mathbb{Q}^{\zeta, h, v}$, introduced in Definition 11 . The choice of $\zeta$ and $v$ is immaterial here and thus it suffices to observe that $\mathbb{Q}^{h}$ is characterized by the property that the processes $\bar{S}^{i}=\left(B^{h}\right)^{-1} S^{i}, i=1,2, \ldots, d$ or, equivalently, the processes

$$
d \bar{S}_{t}^{i}=\left(B_{t}^{h}\right)^{-1}\left(d S_{t}^{i}-h_{t} S_{t}^{i} d t\right)
$$

are $\mathbb{Q}^{h}$-local martingales. Since the default risk cannot be eliminated and thus we deal with an incomplete market model, the uniqueness of a probability measure $\mathbb{Q}^{h}$ fails to hold since the intensity of default times is not uniquely specified under $\mathbb{Q}^{h}$. Therefore, it should be acknowledged that our tentative valuation result presented in Proposition 5 hinges, in fact, on a choice of a particular measure $\mathbb{Q}^{h}$ and possibly also on the hedging error $W-\hat{\pi}$ of a strategy $\varphi$ chosen by the investor.

Proposition 5. The process $\widehat{\pi}=\widehat{\pi}^{\eta, h}(A, R, C, \tau)$ satisfies on $\{t<\widehat{\tau}\}$

$$
\begin{aligned}
\widehat{\pi}_{t} & =\mathbb{E}_{\mathbb{Q}^{h}}\left(\int_{t}^{\widehat{\tau}} D^{\eta}(t, u)\left(\bar{f}_{u}-\eta_{u}\right)\left(\left(W_{u}-\widehat{\pi}_{u}+C_{u}\right)^{+}-\left(W_{u}-\widehat{\pi}_{u}+C_{u}\right)^{-}\right) d u \mid \mathcal{G}_{t}\right) \\
& +\mathbb{E}_{\mathbb{Q}^{h}}\left(\int_{] t, \widehat{\tau}]} D^{\eta}(t, u) d \widetilde{A}_{u}-\int_{t}^{\widehat{\tau}} D^{\eta}(t, u)\left(\bar{c}_{u}-\eta_{u}\right) C_{u} d u \mid \mathcal{G}_{t}\right) \\
& +\mathbb{E}_{\mathbb{Q}^{h}}\left(D^{\eta}(t, \tau) \mathbb{1}_{\{\tau<T\}}\left(Q_{\tau}+\mathbb{1}_{\left\{\tau=\tau_{I}\right\}} L_{I}\left(Q_{\tau_{I}}-C_{\tau_{I}-}\right)^{-}-\mathbb{1}_{\left\{\tau=\tau_{C}\right\}} L_{C}\left(Q_{\tau_{C}}-C_{\tau_{C}-}\right)^{+}\right) \mid \mathcal{G}_{t}\right) \\
& +\mathbb{E}_{\mathbb{Q}^{h}}\left(D^{\eta}(t, \tau) \mathbb{1}_{\{\tau<T\}} \mathbb{1}_{\left\{\tau=\tau_{I}\right\}} L_{I}\left(W_{\tau-}-\widehat{\pi}_{\tau-}+C_{\tau-}\right)^{-} \mid \mathcal{G}_{t}\right) .
\end{aligned}
$$

Proof. The postulated martingale property of $G^{\eta}$ under $\mathbb{Q}^{h}$ yields, for every $t \in[0, T]$ on $\{t<\widehat{\tau}\}$,

$$
\mathbb{E}_{\mathbb{Q}^{h}}\left(G_{T \wedge \tau}^{\eta}-G_{t \wedge \tau}^{\eta} \mid \mathcal{G}_{t}\right)=\mathbb{E}_{\mathbb{Q}^{h}}\left(G_{\tau}^{\eta}-G_{t}^{\eta} \mid \mathcal{G}_{t}\right)=0
$$

and thus we obtain from (48)

$$
\begin{aligned}
\left(B_{t}^{\eta}\right)^{-1} \widehat{\pi}_{t} & =\mathbb{E}_{\mathbb{Q}^{h}}\left(\int_{t}^{\widehat{\tau}}\left(B_{u}^{\eta}\right)^{-1} \psi_{u}^{l} d B_{u}^{l}+\int_{t}^{\widehat{\tau}}\left(B_{u}^{\eta}\right)^{-1} \psi_{u-}^{b} d B_{u}^{b}+\sum_{i=1}^{d} \int_{j t, \widehat{\tau}]}\left(B_{u}^{\eta}\right)^{-1}\left(\xi_{u}^{i} d S_{u}^{i}+\psi_{u}^{i} d B_{u}^{i}\right) \mid \mathcal{G}_{t}\right) \\
& +\mathbb{E}_{\mathbb{Q}^{h}}\left(\int_{] t, \widehat{\tau}]}\left(B_{u}^{\eta}\right)^{-1} d \widetilde{A}_{u}-\int_{t}^{\widehat{\tau}}\left(B_{u}^{\eta}\right)^{-1} \bar{c}_{u} C_{u} d u+\mathbb{1}_{\{\tau<T\}}\left(B_{\tau}^{\eta}\right)^{-1}\left(R_{\tau}+C_{\tau-}\right) \mid \mathcal{G}_{t}\right) \\
& -\mathbb{E}_{\mathbb{Q}^{h}}\left(\int_{t}^{\widehat{\tau}} \eta_{u} \psi_{u}^{l} B_{u}^{l} d u+\int_{t}^{\widehat{\tau}} \eta_{u} \psi_{u}^{b} B_{u}^{b} d u-\int_{t}^{\widehat{\tau}} \eta_{u}\left(B_{u}^{\eta}\right)^{-1} C_{u} d u \mid \mathcal{G}_{t}\right) .
\end{aligned}
$$

We substitute the expressions for differentials and use (3) and the equality $\xi_{u}^{i} S_{u}^{i}+$ $\psi_{u}^{i} B_{u}^{i}=0$ to obtain, for every $t \in[0, T]$ on $\{t<\widehat{\tau}\}$,

$$
\begin{aligned}
\widehat{\pi}_{t} & =\mathbb{E}_{\mathbb{Q}^{h}}\left(\int_{] t, \hat{\tau}]} D^{\eta}(t, u) d \widetilde{A}_{u}+\int_{t}^{\hat{\tau}} D^{\eta}(t, u)\left(\bar{f}_{u}-\eta_{u}\right) F_{u} d u-\int_{t}^{\widehat{\tau}} D^{\eta}(t, u)\left(\bar{c}_{u}-\eta_{u}\right) C_{u} d u \mid \mathcal{G}_{t}\right) \\
& +\mathbb{E}_{\mathbb{Q}^{h}}\left(D^{\eta}(t, \tau) \mathbb{1}_{\{\tau<T\}}\left[Q_{\tau}+\mathbb{1}_{\left\{\tau=\tau_{I}\right\}}\left(L_{I}\left(Q_{\tau_{I}}-C_{\tau_{I}-}\right)^{-}-L_{I} \psi_{\tau_{I}-}^{b} B_{\tau_{I}-}^{b}\right)\right] \mid \mathcal{G}_{t}\right) \\
& -\mathbb{E}_{\mathbb{Q}^{h}}\left(D^{\eta}(t, \tau) \mathbb{1}_{\{\tau<T\}} \mathbb{1}_{\left\{\tau=\tau_{C}\right\}} L_{C}\left(Q_{\tau_{C}}-C_{\tau_{C}-}\right)^{+} \mid \mathcal{G}_{t}\right)
\end{aligned}
$$


where

$$
F_{t}=\psi_{t}^{l} B_{t}^{l}+\psi_{t}^{b} B_{t}^{b}=\left(W_{t}-\widehat{\pi}_{t}+C_{t}\right)^{+}-\left(W_{t}-\widehat{\pi}_{t}+C_{t}\right)^{-}=F_{t}^{+}-F_{t}^{-}
$$

and $W_{t}=W_{t}\left(\varphi, A^{R, C}\right)$. Equation (46) now shows that equality (50) holds on the event $\{t<\widehat{\tau}\}$ for every $t \in[0, T]$.

A priori, some valuation adjustments appearing in the right-hand side in the tentative pricing Formula (50) depend explicitly on the wealth process $W$. However, if we interpret $\eta$ as the deposit rate and we set

$$
\bar{f}_{t}=\eta_{t} \mathbb{1}_{\left\{F_{t}>0\right\}}+\left(\eta_{t}+s_{t}^{f}\right) \mathbb{1}_{\left\{F_{t} \leq 0\right\}}
$$

where $s_{t}^{f}$ is the funding spread of the bank borrowing with respect to the prevailing deposit rate $\eta_{t}$, then it is clear that the first and last terms in the right-hand side of Equation (50) may cancel out provided that the bank's funding spread is "fair", in the sense that the bank's default and recovery are priced correctly by the market.

To examine the last claim in more detail, we denote $Y_{t, s}=D^{\eta}(t, s)\left(W_{s}-\widehat{\pi}_{s}+C_{s}\right)^{-}$ and we compute the net funding/default benefit, $\mathrm{DVA}_{t}^{f,-}-\mathrm{FCA}_{t}^{f}$, which in the present setup is given by the following expression:

$$
J_{t}:=\mathbb{E}_{\mathbb{Q}^{h}}\left(\mathbb{1}_{\{\tau<T\}} \mathbb{1}_{\left\{\tau=\tau_{I}\right\}} L_{I} Y_{t, \tau-} \mid \mathcal{G}_{t}\right)-\mathbb{E}_{\mathbb{Q}^{h}}\left(\int_{t}^{\hat{\tau}} s_{u}^{f} Y_{t, u} d u \mid \mathcal{G}_{t}\right) .
$$

Our next goal is to provide explicit conditions under which the investor's net funding benefit vanishes. To this end, we postulate that $L_{I}$ is a constant and default times $\tau_{I}$ and $\tau_{C}$ are conditionally independent under $\mathbb{Q}^{h}$, with respect to the reference filtration $\mathbb{F}$ (see, for instance, Example 9.1.5 in [34]), with stochastic intensities $\lambda^{I}$ and $\lambda^{C}$, respectively. Then, the intensity of $\tau=\tau_{I} \wedge \tau_{C}$ satisfies $\lambda=\lambda^{I}+\lambda^{C}$ and we can use the standard intensity-based approach to complete the computations of $J_{t}$. In particular, we obtain (see, for instance, Propositions 5.1.1 and 5.1.2 in [34] and Lemma 3.8.1 in [28])

$$
\begin{aligned}
J_{t} & =L_{I} \mathbb{E}_{\mathbb{Q}^{h}}\left(\mathbb{1}_{\{\tau<T\}} \mathbb{1}_{\left\{\tau=\tau_{I}\right\}} Y_{\tau_{I}-} \mid \mathcal{G}_{t}\right)-\mathbb{E}_{\mathbb{Q}^{h}}\left(\int_{t}^{\tau \wedge T} s_{u}^{f} Y_{u} d u \mid \mathcal{G}_{t}\right) \\
& =\mathbb{1}_{\{t<\tau\}} \mathbb{E}_{\mathbb{Q}^{h}}\left(\int_{t}^{T} e^{\Lambda_{t}-\Lambda_{u}} L_{I} \lambda_{u}^{I} Y_{u} d u \mid \mathcal{F}_{t}\right)-\mathbb{1}_{\{t<\tau\}} \mathbb{E}_{\mathbb{Q}^{h}}\left(\int_{t}^{T} e^{\Lambda_{t}-\Lambda_{u}} s_{u}^{f} Y_{u} d u \mid \mathcal{F}_{t}\right)
\end{aligned}
$$

where the hazard process $\Lambda$ of $\tau$ satisfies $\Lambda_{t}=\int_{0}^{t} \lambda_{u} d u$. It is thus clear that if $s_{t}^{f}=L_{I} \lambda_{t}^{I}$ for all $t \in[0, T]$, then $J_{t}=0$ for all $t \in[0, T]$ and thus the equality $\mathrm{FCA}_{t}^{f}=\mathrm{DVA}_{t}^{f,-}$ is satisfied at all times, meaning that, in some circumstances, the funding cost may perfectly offset the benefit at default.

We conclude this paper by establishing a simplified pricing formula in an incomplete market, which does not require specific knowledge of the investor's hedging strategy. Recall that we work under the postulate that for any self-financing strategy the investor's deflated gains process $G^{\eta}$ is a martingale under $\mathbb{Q}^{h}$. We denote

$$
\widehat{\pi}_{t}^{\eta, h}(A):=\mathbb{E}_{\mathbb{Q}^{h}}\left(\int_{] t, T]} D^{\eta}(t, u) d A_{u} \mid \mathcal{G}_{t}\right)
$$

and

$$
Q_{t}^{\eta, h}:=\mathbb{E}_{\mathbb{Q}^{h}}\left(\int_{[t, T]} D^{\eta}(t, u) d A_{u} \mid \mathcal{G}_{t}\right)=\widehat{\pi}_{t}^{\eta, h}(A)+\Delta A_{t} .
$$

The following result shows that the double counting of benefits, which may arise due to a possibility of the bank's default, does not appear if the bank's trading arrangement are modelled adequately, although the net funding/default benefit does not necessarily vanish. 
Proposition 6. Assume that $L_{I}$ is a constant and default times $\tau_{I}$ and $\tau_{C}$ are conditionally independent under $\mathbb{Q}^{h}$ with respect to the reference filtration $\mathbb{F}$. Let the intensity of the bank's default time $\tau_{I}$ under $\mathbb{Q}^{h}$ with respect to the filtration $\mathbb{F}$ equal $\lambda^{I}$ and let for all $t \in[0, T]$

$$
\bar{f}_{t}=\eta_{t} \mathbb{1}_{\left\{F_{t}>0\right\}}+\left(\eta_{t}+L_{I} \lambda_{t}^{I}\right) \mathbb{1}_{\left\{F_{t} \leq 0\right\}}
$$

so that the bank's funding spread equals $s_{t}^{f}=L_{I} \lambda_{t}^{I}$ for all $t \in[0, T]$. Then $\mathrm{FCA}_{t}^{f}=\mathrm{DVA}_{t}^{f,-}$ on the event $\{t<\tau\}$, so that the net funding/default benefit vanishes and $\widehat{\pi}_{t}^{\eta, h}(A, R, C, \tau)$ satisfies the following:

$$
\begin{aligned}
& \widehat{\pi}_{t}^{\eta, h}(A, R, C, \tau)=\widehat{\pi}_{t}^{\eta, h}(A)-\mathbb{E}_{\mathbb{Q}^{h}}\left(\int_{t}^{\widehat{\tau}} D^{\eta}(t, u)\left(\bar{c}_{u}-\eta_{u}\right) C_{u} d u \mid \mathcal{G}_{t}\right) \\
& +\mathbb{E}_{\mathbb{Q}^{h}}\left(D^{\eta}(t, \tau) \mathbb{1}_{\{\tau<T\}}\left(\mathbb{1}_{\left\{\tau=\tau_{I}\right\}} L_{I}\left(Q_{\tau_{I}, h}^{\eta, h}-C_{\tau_{I}-}\right)^{-}-\mathbb{1}_{\left\{\tau=\tau_{C}\right\}} L_{C}\left(Q_{\tau_{C}}^{\eta, h}-C_{\tau_{C^{-}}}\right)^{+}\right) \mid \mathcal{G}_{t}\right)
\end{aligned}
$$

where $Q_{\tau}^{\eta, h}=\widehat{\pi}_{\tau}^{\eta, h}(A)+\Delta A_{\tau}$. Hence the price $\hat{\pi}_{t}^{\eta, h}(A, R, C, \tau)$ admits the following representation:

$$
\widehat{\pi}_{t}^{\eta, h}(A, R, C, \tau)=\widehat{\pi}_{t}^{\eta, h}(A)+\mathrm{DVA}_{t}-\mathrm{CVA}_{t}-\mathrm{LVA}_{t} .
$$

Proof. Using (50) and noting that, under the present assumptions, we have that $J_{t}=0$ for all $t \in[0, T]$, we obtain on the event $\{t<\tau\}$,

$$
\begin{aligned}
& \widehat{\pi}_{t}^{\eta, h}(A, R, C, \tau)=\mathbb{E}_{\mathbb{Q}^{h}}\left(\int_{] t, \hat{\tau}]} D^{\eta}(t, u) d \widetilde{A}_{u}+\int_{t}^{\tau \wedge T} D^{\eta}(t, u)\left(\eta_{u}-\bar{c}_{u}\right) C_{u} d u \mid \mathcal{G}_{t}\right) \\
& +\mathbb{E}_{\mathbb{Q}^{h}}\left(D^{\eta}(t, \tau) \mathbb{1}_{\{\tau<T\}}\left(Q_{\tau}+\mathbb{1}_{\left\{\tau=\tau_{I}\right\}} L_{I}\left(Q_{\tau_{I}}-C_{\tau_{I}-}\right)^{-}-\mathbb{1}_{\left\{\tau=\tau_{C}\right\}} L_{C}\left(Q_{\tau_{C}}-C_{\tau_{C^{-}}}\right)^{+}\right) \mid \mathcal{G}_{t}\right) .
\end{aligned}
$$

If we postulate that $Q=Q^{\eta, h}$, then to derive the desired representation for $\widehat{\pi}_{t}^{\eta, h}(A, R, C, \tau)$, it suffices to proceed as in the proof of Proposition 2.

It is worth noting that, under the assumptions of Proposition 5, the ex-dividend selling price $\widehat{\pi}^{\eta, h}(A, R, C, \tau)$ is independent of the wealth process $W$ of a strategy $\varphi$ chosen by the investor. Observe also that the quantities $\mathrm{DVA}_{t}$ and $\mathrm{CVA}_{t}$ are always non-negative. In contrast, the sign of $\mathrm{LVA}_{t}$ depends on the relationship between $\eta$ and $\bar{c}$, as well as on the sign of $C$.

\section{Conclusions}

In this paper, we have derived the adjusted price of a derivative contract in the presence of credit risk (default of counterparties) and with funding and collateral costs using two approaches, concerned that they may not lead to the same valuation formula.

The starting point was the "clean price" of the contract that is computed in (1) in the absence of credit risk or any funding and collateral costs. We then incorporated these additional risk factors and costs in the cash flows under the "adjusted cash flow" approach, which resulted in a nonlinear valuation formula (Proposition 1). We illustrated that using additive valuation adjustments (XVAs) to express the adjusted price, as in (7), ignored the interactions between the terms. Instead, we showed that asymmetric borrowing and lending rates for the funding sources, as well as asymmetric repo rates for long and short positions, naturally led to a nonlinear valuation formula (Proposition 3) that can be solved numerically via nonlinear BSDEs (Proposition 4).

The second approach used to derive the adjusted price of the contract was the "replication approach", presented in Section 3. A closed-form solution to the valuation problem was presented in Corollary 1, and can be computed explicitly for several cases of interest, while the general nonlinear valuation formula was proved in Theorem 2.

Finally, we are able to answer in the affirmative the question that motivated this research: the "adjusted cash flow" and the "replication" approaches lead to the same 
valuation formula, and we prove this in Corollary 3 under the specific and reasonable assumption that external costs, such as DVA2 terms, are not considered. This reconciles the views of academics and practitioners on nonlinear valuation formulae in the current multi-curve environment.

Author Contributions: D.B., C.B., M.F., A.P. and M.R. have been involved at all stages. All authors have read and agreed to the published version of the manuscript.

Funding: The research of Damiano Brigo and Marek Rutkowski was supported by the EPSRC Mathematics Platform Grant EP/I019111/1 Mathematical Analysis of Funding Costs at Imperial College London. The research of M. Rutkowski was supported by the Australian Research Council Discovery Project DP200101550.

Informed Consent Statement: Not applicable.

Data Availability Statement: Not applicable.

Conflicts of Interest: The authors declare no conflict of interest.

\section{References}

1. Bichuch, M.; Capponi, A.; Sturm, S. Arbitrage-free XVA. Math. Financ. 2018, 28, 582-620. [CrossRef]

2. Brigo, D.; Francischello, M.; Pallavicini, A. Analysis of nonlinear valuation equations under credit and funding effects. In Innovations in Derivatives Markets, Fixed Income Modeling, Valuation Adjustments, Risk Management, and Regulation; Glau, K., Grbac, Z., Scherer, M., Zagst, R., Eds.; Springer Proceedings in Mathematics and Statistics; Springer: Berlin/Heidelberg, Germany, 2016; Volume 165, pp. 37-52.

3. Brigo, D.; Francischello, M.; Pallavicini, A. Nonlinear valuation under credit, funding, and margins: existence, uniqueness, invariance, and disentanglement. Eur. J. Oper. Res. 2019, 274, 788-805. [CrossRef]

4. Brigo, D.; Pallavicini, A. Nonlinear consistent valuation of CCP cleared or CSA bilateral trades with initial margins under credit, funding and wrong-way risks. J. Financ. Eng. 2014, 1, 1450001. [CrossRef]

5. Crépey, S. Bilateral counterparty risk under funding constraints-Part I: Pricing. Math. Financ. 2015, 25, 1-22. [CrossRef]

6. Crépey, S. Bilateral counterparty risk under funding constraints-Part II: CVA. Math. Financ. 2015, 25, 23-50. [CrossRef]

7. Nie, T.; Rutkowski, M. Fair bilateral prices in Bergman's model with exogenous collateralization. Int. J. Theor. Appl. Financ. 2015, 18, 1550048. [CrossRef]

8. Nie, T.; Rutkowski, M. A BSDE approach to fair bilateral pricing under endogenous collateralization. Financ. Stoch. 2016, 20, 855-900. [CrossRef]

9. Nie, T.; Rutkowski, M. Fair bilateral prices under funding costs and exogenous collateralization. Math. Financ. 2018, 28, 621-655. [CrossRef]

10. Backwell, A.; Macrina, A.; Schlögl, E.; Skovm, D. Term Rates, Multicurve Term Structures and Overnight Rate Benchmarks: A RollOver Risk Approach. Working Paper. 2021. Available online: https:/ / papers.ssrn.com/sol3/papers.cfm?abstract_id=3399680 (accessed on 13 December 2021).

11. Berndt, A.; Duffie, D.; Zhu, Y. Across-the-Curve Credit Spread Indices. Working Paper. 2020. Available online: www.gsb.stanford. edu/faculty-research/working-papers/across-curve-credit-spread-indices (accessed on 13 December 2021 ).

12. Gellert, K.; Schlögl, E. Short Rate Dynamics: A Fed Funds and SOFR Perspective. FIRN Research Paper. 2021. Available online: https://papers.ssrn.com/sol3/papers.cfm?abstract_id=3763589 (accessed on 13 December 2021).

13. Lyashenko, A.; Mercurio, F. Looking Forward to Backward Looking Rates; Working Paper; Quantitative Risk Management, Inc.: Chicago, IL, USA, 2019.

14. Mercurio, F. Modern LIBOR market models: Using different curves for projecting rates and for discounting. Int. J. Theor. Appl. Financ. 2010, 13, 113-137. [CrossRef]

15. Mercurio, F. A Simple Multi Curve Model for Pricing SOFR Futures and Other Derivatives; Working Paper; Bloomberg LP: New York, NY, USA, 2018.

16. Piterbarg, V. Funding beyond discounting: Collateral agreements and derivative pricing. Risk Magazine, 3 February 2010; pp. 97-102.

17. CME Group. CME Group Announces Record SOFR Futures Volume and Open Interest. 2021. Available online: www.prnewswire. com/news-releases/cme-group-announces-record-sofr-futures-volume-and-open-interest-301405903.html (accessed on 13 December 2021 ).

18. Financial Conduct Authority. Announcements on the End of LIBOR. 2021. Available online: www.fca.org.uk/news/pressreleases/announcements-end-libor (accessed on 13 December 2021).

19. Federal Reserve Bank of New York. Additional Information about Reference Rates Administered by the New York Fed. 2021. Available online: www.newyorkfed.org/markets/reference-rates/additional-information-about-reference-rates (accessed on 13 December 2021). 
20. Federal Reserve Bank of New York. Secured Overnight Financing Rate Data. 2021. Available online: www.newyorkfed.org/ markets/reference-rates/sofr (accessed on 13 December 2021).

21. ISDA. Interest Rate Benchmarks Review: Full Year 2019 and the Fourth Quarter of 2019. January 2020. Available online: www.isda.org/a/W5LTE/Interest-Rate-Benchmarks-Review-Full-Year-2019-and-Q4-2019.pdf (accessed on 13 December 2021).

22. Pallavicini, A.; Perini, D.; Brigo, D. Funding Valuation Adjustment: A Consistent Framework Including CVA, DVA, Collateral, Netting Rules and Re-Hypothecation. arXiv 2011, arXiv:1112.1521.

23. Pallavicini, A.; Perini, D.; Brigo, D. Funding, Collateral and Hedging: Uncovering the Mechanics and the Subtleties of Funding Valuation Adjustments. DVA Collat. Netting Rules Re-Hypothecation 2011, http://dx.doi.org/10.2139/ssrn.2161528. [CrossRef]

24. Bielecki, T.R.; Rutkowski, M. Valuation and hedging of contracts with funding costs and collateralization. SIAM J. Financ. Math. 2015, 6, 594-655. [CrossRef]

25. Bielecki, T.R.; Cialenco, I.; Rutkowski, M. Arbitrage-free pricing of derivatives in nonlinear market models. Probab. Uncertain. Quant. Risk 2018, 3, 2. [CrossRef]

26. Bielecki, T.R.; Cialenco, I.; Iyigunler, I. Collateralized CVA valuation with rating triggers and credit migrations. Int. J. Theor. Appl. Financ. 2013, 16, 1350009. [CrossRef]

27. Bielecki, T.R.; Jeanblanc, M.; Rutkowski, M. PDE approach to valuation and hedging of credit derivatives. Quant. Financ. 2005, 5, 257-270. [CrossRef]

28. Bielecki, T.R.; Jeanblanc, M.; Rutkowski, M. Credit Risk Modeling; Osaka University Press: Osaka, Japan, 2009.

29. Hull, J.; White, A. The FVA debate. Risk Magazine, 13 July 2012, pp. 83-85.

30. Brigo, D.; Capponi, A.; Pallavicini, A.; Papatheodorou, V. Pricing counterparty risk including collateralization, netting rules, re-hypothecation and wrong-way risk. Int. J. Theor. Appl. Financ. 2013, 16, 1350007. [CrossRef]

31. Bormetti, G.; Brigo, D.; Francischello, M.;Pallavicini, A. Impact of multiple curve dynamics in credit valuation adjustments under collateralization. Quant. Financ. 2018, 18, 31-44. [CrossRef]

32. Brigo, D.; Buescu, C.; Rutkowski, M. Funding, repo and credit inclusive valuation as modified option pricing. Oper. Res. Lett. 2017, 45, 665-670. [CrossRef]

33. Brigo, D.; Masetti, M. Risk neutral pricing of counterparty risk. In Counterparty Credit Risk Modelling: Risk Management, Pricing and Regulation; Pykhtin, M., Ed.; Risk Books: London, UK, 2005.

34. Bielecki, T.R.; Rutkowski, M. Credit Risk: Modeling, Valuation and Hedging; Springer: Berlin/Heidelberg, Germany, 2002.

35. Elliott, R.J.; Jeanblanc, M.; Yor, M. On models of default risk. Math. Financ. 2000, 10, 179-195. [CrossRef]

36. Brigo, D.; Buescu, C.; Morini, M. Counterparty risk pricing: Impact of closeout and first to default times. Int. J. Theor. Appl. Financ. 2012, 15, 1250039. [CrossRef]

37. Dur, ; C.; Rutkowski, M. CVA under alternative settlement conventions and with systemic risk. Int. J. Theor. Appl. Financ. 2013, 16, 1350039. [CrossRef]

38. Crépey, S.; Bielecki, T.R.; Brigo, D. Counterparty Risk and Funding: A Tale of Two Puzzles; Chapman \& Hall/CRC Financial Mathematics Series; CRC Press: Boca Raton, FL, USA, 2014.

39. Andersen, L.; Duffie, D.; Song, Y. Funding valuation adjustments. J. Financ. 2019, 74, 145-192. [CrossRef]

40. Burgard, C.; Kjaer, M. Partial differential equation representations of derivatives with bilateral counterparty risk and funding costs. J. Credit. Risk 2011, 7, 75-93. [CrossRef]

41. Crépey, S.; Gerboud, R.; Grbac, Z.; Ngor, N. Counterparty risk and funding: The four wings of TVA. Int. J. Theor. Appl. Financ. 2013, 16, 1350006. [CrossRef]

42. Biffis, E.; Blake, D.; Pitotti, L.; Sun, A. The cost of counterparty risk and collateralization in longevity swaps. J. Risk Insur. 2016, 83, 387-419. [CrossRef]

43. Baviera, R.; La Bua, G.; Pellicioli, P. CVA with wrong-way risk in the presence of early exercise. In Innovations in Derivatives Markets; Glau, K., Grbac, Z., Scherer, M., Zagst, R., Eds.; Springer Proceedings in Mathematics and Statistics; Springer: Berlin/Heidelberg, Germany, 2016; Volume 165, pp. 103-116.

44. Brigo, D.; Capponi, A.; Pallavicini, A. Arbitrage-free bilateral counterparty risk valuation under collateralization and application to Credit Default Swaps. Math. Financ. 2014, 24, 125-146. [CrossRef]

45. El Karoui, N.; Peng, S.; Quenez, M.C. Backward stochastic differential equations in finance. Math. Financ. 1997, 7, 1-71. [CrossRef]

46. El Karoui, N.; Quenez, M.C. nonlinear pricing theory and backward stochastic differential equations. In Financial Mathematics; Runggaldier, W.J., Ed.; Lecture Notes in Mathematics 1656; Springer: Berlin/Heidelberg, Germany, 1997; pp. $191-246$.

47. Crépey, S.; Song, S. Counterparty risk and funding: Immersion and beyond. Financ. Stochastics 2016, 20, 901-930. [CrossRef]

48. Dumitrescu, R.; Grigorova, M.; Quenez, M.C.; Sulem, A. BSDEs with default jump. In Computation and Combinatorics in Dynamics, Stochastics and Control ; Celledoni, E., Di Nunno, G., Ebrahimi-Fard, K., Munthe-Kaas, H., Eds.; Springer: Cham, Switzerland , 2018; Volume 13, pp. 233-263.

49. Peng, S.; Xu, X. BSDEs with Random Default Time and Their Applications to Default Risk. arXiv 2009, arXiv:0910.2091.

50. Carbone, R.; Ferrario, B.; Santacroce, M. Backward stochastic differential equations driven by càdlàg martingales. Theory Probab . Its Appl. 2015, 52, 304-314. [CrossRef]

51. Nie, T.; Rutkowski, M. Reflected BSDEs and doubly reflected BSDEs driven by RCLL martingales. Stochastics Dyn. 2021, 2021, 2250012. [CrossRef] 